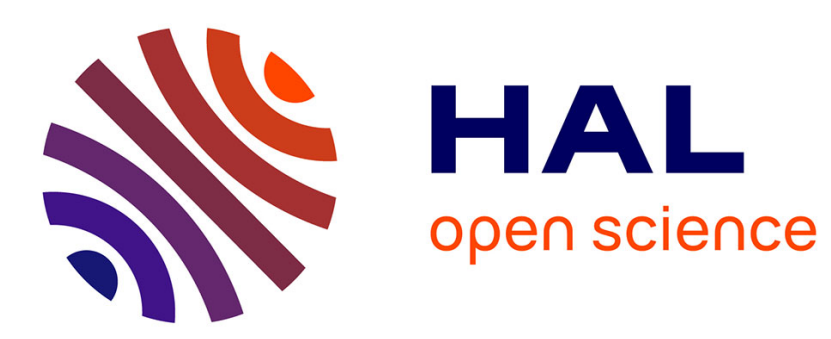

\title{
Apport de la sûreté de fonctionnement à l'analyse spatialisée du risque inondation
}

Michaël Gonzva, Bruno Barroca, Serge Lhomme, Pierre-Etienne Gautier, Youssef Yd Diab

\section{- To cite this version:}

Michaël Gonzva, Bruno Barroca, Serge Lhomme, Pierre-Etienne Gautier, Youssef Yd Diab. Apport de la sûreté de fonctionnement à l'analyse spatialisée du risque inondation. Revue Internationale de Géomatique, 2016, 26 (3), pp.329-361. 10.3166/rig.2016.00003 . hal-01676222

\section{HAL Id: hal-01676222 \\ https://hal.science/hal-01676222}

Submitted on 5 Jan 2018

HAL is a multi-disciplinary open access archive for the deposit and dissemination of scientific research documents, whether they are published or not. The documents may come from teaching and research institutions in France or abroad, or from public or private research centers.
L'archive ouverte pluridisciplinaire HAL, est destinée au dépôt et à la diffusion de documents scientifiques de niveau recherche, publiés ou non, émanant des établissements d'enseignement et de recherche français ou étrangers, des laboratoires publics ou privés. 


\title{
Apport de la sûreté de fonctionnement à l'analyse spatialisée du risque inondation
}

\author{
Michaël Gonzva $^{1,3}$, Bruno Barroca ${ }^{1}$, Serge Lhomme ${ }^{2}$, \\ Pierre-Étienne Gautier ${ }^{3}$, Youssef Diab ${ }^{1,4}$
}

1. Université Paris-Est Marne-la-Vallée, Département Génie Urbain, Lab'Urba. 6-8 avenue Blaise Pascal, Cité Descartes, Champs-sur-Marne, 77455

Marne-La-Vallée Cedex 2, France.

\{michael.gonzva,bruno.barroca,youssef.diab\}@u-pem.fr

2. Université Paris-Est Créteil, Département de Géographie, Lab’Urba.

Campus Centre - 61, avenue du Général de Gaulle, 94010 Créteil Cedex, France.

serge.lhomme@u-pec.fr

3. SYSTRA - Direction Innovation.

72, rue Henry Farman, 75015 Paris, France.

\{mgonzva,pegautier\}@systra.com

4. Université Paris-Est, École des Ingénieurs de la Ville de Paris.

78-80 rue Rébéval, 75019 Paris, France.

youssef.diab@eivp-paris.fr

RÉSUMÉ. Les systèmes techniques urbains sont soumis à des enjeux importants, notamment climatiques et territoriaux, qui affectent leur fonctionnement. Ces enjeux rendent alors les systèmes techniques vulnérables aux aléas naturels. Or, les méthodologies usuellement mobilisées pour l'analyse de la vulnérabilité restent essentiellement aléa-centrées, c'est-à-dire qu'elles se focalisent sur l'aléa en tant que phénomène physique et sur les dommages directs qu'il provoque sur le système. Cette approche semble peu ou pas indiquée lorsque le système étudié est caractérisé par une importante complexité, par une forte interconnexion avec d'autres systèmes techniques et par des dynamiques de défaillances multiples et simultanées. Dans cet article, nous nous intéressons à l'analyse de la vulnérabilité des systèmes de transport guidé face au risque d'inondation. Cette recherche porte sur une approche innovante d'analyse des risques qui associe à la fois des méthodes issues de la sûreté de fonctionnement et des méthodes issues de la géomatique à l'aide de l'information géographique. L'approche est volontairement système-centrée pour concevoir une méthodologie apte à caractériser les atteintes directes et indirectes au système. L'enjeu global est de mettre en exergue l'apport d'une telle méthode pour la gestion des risques urbains, en contribuant ainsi à la résilience globale des villes face à des risques d'inondation.

Revue internationale de gomatique $-\mathrm{n}^{0} 3 / 2016,1-33$ 
ABSTRACT. The urban technical systems are subjected to major stakes, such as climatic and territorial issues, which affect their functioning. These stakes can make the technical systems vulnerable to natural hazards. But, the traditional methodologies used for vulnerability analyses are hazard-oriented. They are focused on the hazard as a physical process and on the direct damages to the system. This approach is found to be unsuitable for analyzing systems with a high level of complexity, interconnectedness and linkages with other urban technical systems, and with many and varied failures processes. This paper analyzes the vulnerability of guided transport systems facing flood risk. This research focuses on an innovative approach of risks analysis that associates safety and dependability methods with methods from geomatics. The geographical information allows this combination between these two very different types of methods. The chosen approach is system-oriented in order to elaborate a methodology for characterizing any direct and indirect damages to the system. The global goal is to highlight the contribution of this innovative approach for the urban risk management, and so improve the resilience of cities facing of flood risks.

MOTS-CLÉS : système technique urbain, risque naturel, modèle fonctionnel, modèle relationnel KEYWORDS: urban technical system, natural risk, functional model, relational model

DOI:10.3166/RIG.x.1-33 (c) 2016 Lavoisier

\section{Introduction}

La conception d'un système technique en milieu urbain ne peut aujourd'hui s'affranchir d'un nombre toujours plus important d'enjeux de plusieurs ordres : des enjeux climatiques, géographiques ou encore fonctionnels. Les enjeux climatiques témoignent de la sensibilité constatée des systèmes techniques urbains à des conditions météorologiques intenses : pluviométrie, variations de températures, sismicité, etc. Les enjeux géographiques révèlent les conditions dans lesquelles un système technique s'insère sur un territoire donné : usage des sols, topographie, types de terrain, etc. Les enjeux fonctionnels, quant à eux, visent à établir des liens entre les usages, les besoins d'une part et le service fourni d'autre part en adaptant notamment les modes d'exploitation du système : mode nominal, modes dégradés... Dans le cas d'un système de transport public guidé, qui est le système technique urbain étudié dans cet article, la prépondérance de ces enjeux est pleinement vérifiée. Du point de vue climatique, la vulnérabilité empirique des systèmes de transport guidé est très régulièrement avérée lorsqu'ils sont affectés par des aléas naturels d'intensité variable (Oslakovic Stipanovic et al., 2013) et se traduit par des incidents et accidents. Du point de vue géographique, les caractéristiques du territoire dans lequel s'implante un système de transport ne sont pas anodines sur son exposition : une implantation sur un terrain à fort relief ou au contraire très plat n'aura pas la même incidence sur l'intensité et les potentiels impacts d'aléas naturels. Enfin, du point de vue fonctionnel, les besoins en mobilité des usagers doivent être assurés par un service adapté du système de transport quelles que soient les conditions climatiques ou géographiques. Ainsi, la conception d'un système technique oscille entre cet ensemble d'enjeux dont la coexistence apparaît difficile. 
La prépondérance de ces enjeux dans la conception et l'exploitation des systèmes techniques s'inscrit, de surcroît, dans une forte perspective d'intensification. La part de la population mondiale qui vit dans des zones urbaines est en pleine croissance ce qui a nécessairement des conséquences importantes sur les besoins en déplacements urbains et interurbains; dans ce contexte, freiner la mobilité ne peut être une option (Commission Européenne, 2011). Les changements climatiques semblent, de plus, avoir un rôle amplificateur sur les risques naturels qui doivent donc être réexaminés en fonction de cette nouvelle donne climatique (Quenault, 2013b). Parmi ces risques naturels, le risque d'inondation apparaît comme l'un des plus catastrophiques car le plus fréquent parmi toutes les catastrophes naturelles. En France, un habitant sur quatre et un emploi sur trois sont en effet potentiellement exposés au risque d'inondation (Ministère de l'Écologie du Développement Durable et de l'Énergie, 2012). Ainsi, dans ces conditions, le besoin en méthodologies d'analyse des risques qui affectent les systèmes techniques urbains apparait comme manifeste, sinon indispensable.

Ces méthodologies d'analyses de risque, pour être pertinentes et intégrées, se doivent d'être inscrites spatialement. Autrement dit, le risque doit être étudié pour et sur un territoire donné. Cette réflexion prônant une articulation entre le risque et le territoire bénéficie d'une littérature scientifique internationale importante (Scarwell, Laganier, 2004; Muller-Mahn, 2012; Veyret et al., 2004 ; Beucher, 2008). De manière concise, les études géographiques du risque doivent tenir compte de la relation riche et complexe entre ces deux éléments, risque et territoire, sous peine de ne prendre en compte qu'une vision partielle du territoire, à savoir son rôle de support du risque (November, 2002). L'essor de la géomatique dans les années 1990 en tant que champ technique et scientifique dérivant de la géographie et de l'informatique dont un des objectifs est le développement de méthodes pour représenter, analyser et simuler l'espace géographique (Bucher, Le Ber, 2012) a permis d'importants progrès dans l'inscription spatiale du risque.

Au sein de la recherche scientifique, depuis le milieu des années 2000, le concept de résilience véhicule une nouvelle manière d'analyser le risque, particulièrement lorsqu'il affecte une zone urbaine. En effet, la résilience apparaît comme toute indiquée dans une analyse des risques touchant les systèmes urbains dans la mesure où son approche est systémique, offrant alors l'opportunité d'une vision globale des interactions et rétroactions entre le système technique lui-même et son environnement. Les multiples échelles, spatiales et temporelles, au sein desquelles ces interactions se déroulent sont aussi prises en compte car le concept de résilience couvre à la fois les mesures préventives pré-catastrophes et les stratégies post-catastrophes ${ }^{1}$ (Bruneau et al., 2003). Ces interactions et rétroactions, à l'échelle d'un système, bénéficient justement d'un grand nombre de méthodes d'analyses éprouvées dans des domaines techniques industriels différents, en France comme à l'international et depuis plusieurs dizaines d'années qui sont regroupées, pour certaines, sous l'expression de «sûreté de fonctionnement ». De manière succincte, la sûreté de fonctionnement est l'aptitude

1. "In the context of hazards, the concept can be thought of as spanning both pre-event measures that seek to prevent hazard-related damage and losses and post-event strategies designed to cope with and minimize disaster impacts." 
d'un système à assurer ses fonctions et pour cela, elle s'intéresse aux défaillances et pannes potentielles des composants qui constituent ce système. Or, l'approche analytique de la sûreté de fonctionnement peine à prendre en compte ces interactions entre des systèmes toujours plus dépendants et fortement imbriqués, nécessitant par là même un niveau supérieur pour leur étude qui passe alors de celui de «systèmes complexes » (Le Moigne, 1999) à celui de «systèmes de systèmes interdépendants » (Kröger, 2008). Dans ce contexte, la résilience urbaine, fondamentalement systémique et mobilisée dans de très nombreux domaines aussi divers que variés (Francis, Bekera, 2014), est alors définie comme la capacité de la ville à absorber une perturbation puis à récupérer ses fonctions à la suite de celle-ci (Lhomme et al., 2010). La gestion des risques est alors ici considérée comme un point d'entrée pour l'opérationnalisation et la quantification de la résilience (Mitchell, Harris, 2012).

Cet article porte sur une innovation méthodologique qui consiste à croiser des méthodes issues de deux domaines scientifiques, la sûreté de fonctionnement et la géomatique, pour l'élaboration d'un outil méthodologique d'évaluation de la vulnérabilité. Par le rapprochement de ces méthodes, l'article montre qu'il est possible de se servir d'une approche pour construire l'autre bien que les approches en question soient issues de domaines distincts. Les méthodes utilisées sont respectivement l'analyse fonctionnelle et l'analyse des modes de défaillances et de leurs effets d'une part, qui sont croisées avec les bases de données géographiques et systèmes d'information géographique d'autre part. L'objectif de cette méthode est la caractérisation de la vulnérabilité d'un système de transport guidé soumis à un risque d'inondation à partir d'une spatialisation d'un modèle fonctionnel et dysfonctionnel du système. L'enjeu global est de mettre en exergue l'apport d'une telle méthode pour la gestion des risques urbains, en contribuant ainsi globalement à la résilience des villes face à des risques d'inondation.

\section{2. État de l'art des méthodes pour l'analyse des risques affectant les systèmes techniques urbains}

Cette première partie aborde quelques méthodes existantes pour l'analyse des risques naturels qui touchent les systèmes techniques urbains. De façon générale, ces méthodes étudient les interactions qui existent entre aléa naturel, système technique et information géographique. Les risques sont alors ici étudiés autour de ce triptyque dans le but de caractériser un modèle de gestion territorialisée de ces risques naturels affectant les systèmes techniques en milieu urbain (FIGURE 1). De fait, ce triptyque ne peut offrir une vision complète des axes d'amélioration de la gestion des risques urbains dans la mesure où il ne considère pas toutes les composantes de la ville, uniquement ses systèmes techniques. Or, l'amélioration de la résilience urbaine, pour laquelle l'amélioration de la gestion des risques est un enjeu sous-jacent, reste un problème très vaste qui intersecte de nombreux domaines de compétences (Toubin, 2014). Cependant, l'identification fréquente de certains systèmes techniques urbains, en particulier les systèmes de transport, comme des infrastructures dites critiques ou essentielles (Robert, Morabito, 2009 ; Bouchon, 2006 ; O’Rourke, 2007) pour le fonc- 
tionnement des villes démontre le rôle prépondérant que peuvent jouer ces systèmes dans l'amélioration de la résilience urbaine (Lhomme, 2012). Ainsi, ce triptyque est volontairement abordé autour de dimensions matérielles, structurelles, fonctionnelles. Les autres dimensions urbaines - sociale, culturelle, architecturale, environnementale, économique, de gouvernance... - en lien avec le fonctionnement des systèmes techniques ne sont donc pas traitées dans cette recherche bien qu'elles prennent part dans l'amélioration de la gestion des risques urbains et, conséquemment, à la construction de la résilience urbaine.

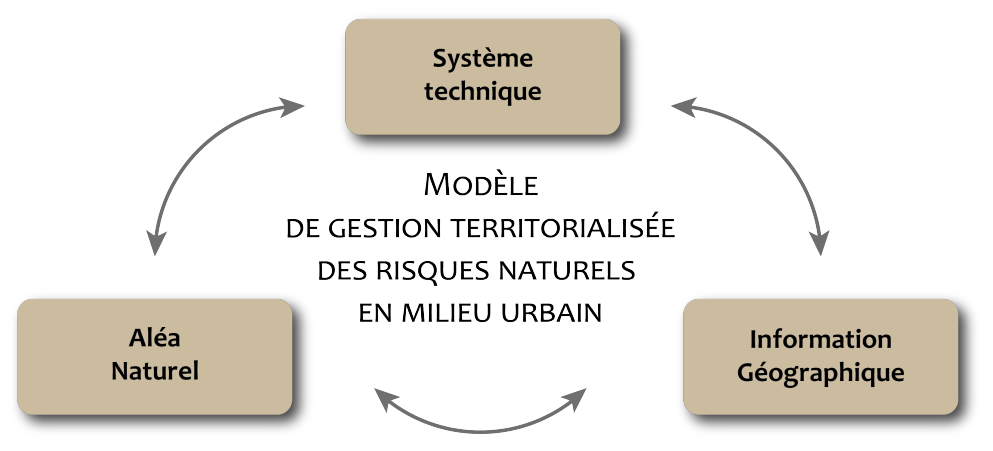

Figure 1. Modèle de gestion territorialisée des risques naturels en milieu urbain centré autour du triptyque aléa naturel-système technique-information géographique

Les objets étudiés dans la suite de cet article sont définis de la façon suivante. Les systèmes techniques urbains peuvent être définis par le service rendu, par leur organisation c'est-à-dire les aspects fonctionnels, et par l'infrastructure qui représente les aspects physiques nécessaires à la réalisation du service (Blancher, 1998). Cette définition française s'accorde tout à fait avec la définition américaine d'une infrastructure qui est caractérisée comme un ensemble d'installations et de services nécessaires au fonctionnement de la société (ASCE, 2009). Un système de transport guidé, exemple type de système technique urbain, peut se définir comme un système dont les véhicules suivent, sur tout ou partie de leur parcours, une trajectoire déterminée. Sont donc inclus les métros, les tramways, les lignes ferroviaires interurbaines et les lignes ferroviaires à grande vitesse. De plus, l'aléa hydrologique pris en compte est générique, c'est-à-dire qu'il regroupe les phénomènes depuis les évènements pluvieux à période de retour faible jusqu'aux crues majeures. La cinétique de l'aléa, lente ou rapide, n'est pas intégrée dans le modèle présenté dans la mesure où la recherche étudie l'impact de l'aléa, c'est-à-dire le risque généré sur le système, et non l'aléa lui-même. L'ensemble de ce travail de recherche s'inscrit donc dans une approche spatiale du risque et a fortiori de la vulnérabilité, générée par ce risque, des systèmes de transport guidé. 


\subsection{Gestion des risques et apports de l'information géographique}

\subsubsection{Une approche aléa-centrée et spatialisée}

Les risques, a fortiori urbains, entretiennent une relation manifeste avec les espaces qu'ils affectent. La question fondamentale n'est alors plus de prouver l'existence de cette connexion entre ces deux éléments mais plutôt d'en déterminer les tenants et les aboutissants. L'émergence de cette relation et le besoin d'en identifier les caractéristiques, du moins d'en dresser les contours, n'est pas récente; dès les années 1980, parmi les changements nouveaux soulevés par Ulrich Beck qui s'opéraient au sein des risques, l'idée de circonscription spatiale apparait déjà. En effet, les risques provoquent dorénavant des dommages irréversibles, irréparables qui n’ont alors plus de limites, ni spatiales, ni temporelles (Beck, 2001). Le lien entre risque et territoire est donc apparu incidemment avec l'évolution des risques eux-mêmes. Dès lors, l'étude de cette relation complexe entre les risques et les espaces qu'ils touchent s'est faite selon plusieurs axes. Tout d'abord un premier axe d'étude visant à définir la spatialité des risques selon laquelle quelques risques semblent coller à certains lieux malgré des mesures mises en place (November, 2006). Un autre axe d'étude de la relation risque-territoire considère la ou les dynamique(s) spatiale(s) des risques. Ces dynamiques sont distinguées selon plusieurs catégories : certains auteurs parlent par exemple de risques-sites, de risques-transports dans le domaine des risques technologiques (Glatron, 1997), de risques-territorialisés, de risques-diffus ou de risques-réseaux selon leur degré d'ancrage à l'espace (Galland, 2003). La localisation de la racine spatiale du risque (D'Ercole, Metzger, 2009) est donc un élément nécessaire dans la compréhension des effets du risque, à savoir les vulnérabilités qu'il induit. Car, de l'approche spatiale du risque découle l'approche spatiale de la vulnérabilité des systèmes urbains vus en tant qu'enjeux. Dès lors, la vulnérabilité territoriale est approchée au travers des enjeux ou espaces-enjeux, évaluée selon la distribution spatiale des enjeux majeurs - populations, équipements sensibles, économie urbaine, etc. - et se transmet alors de lieux particuliers vers des ensembles territoriaux plus vastes (D’Ercole, Metzger, 2009). Ainsi, la compréhension de la dimension spatiale des risques ne peut prendre comme base qu'un «territoire support » car la vision obtenue serait trop parcellaire et occulterait les dynamiques territoriales engendrées par les situations à risque (November et al., 2008). La proximité entre risque et espace a donc immanquablement conduit à penser la gestion des risques comme un processus territorialisé centré autour de systèmes à enjeux élevés. De ce fait, le raisonnement sur le risque s'est fait par «couches », c'est-à-dire qu'à une couche contenant des informations relatives aux enjeux présents sur un espace est croisée avec une autre couche d'informations en lien avec un aléa. Le croisement des informations de ces deux couches aboutit alors à une évaluation du risque subi par ces enjeux, c'est-à-dire que leur vulnérabilité face à l'aléa étudié est mesurée par rapport aux dommages directs qu'ils subissent. Cette approche par l'aléa amène alors à une gestion des risques dont la pensée de fond consiste à réduire les conséquences des dommages potentiels par une protection au sens strict des enjeux. Cette approche a longtemps prédominé dans la gestion des risques. Dès lors, la complexité du concept de risque s'est re- 
trouvée réduite à la seule dimension physique des impacts directs, ce risque étant par conséquent circonscrit par le zonage de l'aléa. Cette approche aléa-centrée n'est pas critiquée en tant que telle car elle répond très bien aux problématiques de certains acteurs de la ville tels que des aménageurs ayant besoin de prescriptions spécifiques relatives aux constructions et activités possibles selon un aléa (Quenault, 2013a). Cependant, là où l'approche aléa-centrée semble présenter des limites, c'est lorsque les enjeux majeurs en question présentent des caractéristiques fortes de complexité et de fonctionnement systémique. Il est alors opportun de se tourner vers une approche système-centrée et territorialisée dans laquelle la réflexion est toujours dépendante de l'aléa qui reste un paramètre d'entrée pour l'analyse de risque mais s'oriente vers des «aléas localisés » et s'intéresse alors à des territoires plutôt que des processus physiques (Weichselgartner, 2004).

\subsubsection{Une approche système-centrée et territorialisée}

L'approche par les enjeux a guidé les premières études de vulnérabilité territoriale (Délégation aux Risques Majeurs, 1990). Les pays anglo-saxons parlent quant à eux plutôt de lifeline (Cutter et al., 2000) pour identifier ces infrastructures-clés pour un territoire. Ces enjeux ou lifelines incluent, en plus des éléments stratégiques usuels tels que des équipements majeurs de transport ou de soins par exemple, que depuis récemment des éléments plus larges liés au fonctionnement et au développement urbain : patrimoine urbain, économie... (D'Ercole, Metzger, 2009). Les lifelines sont très similaires au concept d'infrastructures urbaines critiques, qualifiées ainsi au vu de la dépendance croissante des populations vis-à-vis de celles-ci (La Porte, 2006). Ces infrastructures critiques qui constituent l'épine dorsale des sociétés modernes ${ }^{2}$ (Ventura et al., 2010) et dont les caractéristiques principales sont bien définies (Kröger, 2008) sont collectivement identifiées sur le plan international. Les transports, publics et de marchandise, font partie de ces infrastructures à très fort enjeu. Or, les infrastructures critiques sont de plus en plus complexes, vulnérables et dont la portée s'étend à des échelles supranationales requérant alors des approches innovantes pour réduire les risques de perturbations des services qu'ils fournissent (Cagno et al., 2008 ; Rinaldi et al., 2001). Leur complexité propre est réhaussée globalement du fait de l'existence de relations d'interdépendance entre ces infrastructures critiques (Toubin et al., 2012) obligeant à un changement d'échelle pour analyser les composants d'un système (échelle fine) puis les relations entre les systèmes (échelle plus large) (Serre et al., 2016). Une manière d'étudier les infrastructures critiques consiste alors à les considérer comme des systèmes socio-techniques complexes (Ventura et al., 2010) présentant les caractéristiques principales suivantes : une importante diversité d'éléments, un grand nombre d'interactions dynamiques entre ces éléments et une forte variabilité imprévisible (Saurin, Sosa Gonzalez, 2013). Les dommages provoqués par l'occurrence d'un aléa sur ces infrastructures critiques sont, de fait, difficiles à appréhender dans la mesure où, en plus des dommages directs aux éléments impactés, des dommages indirects dûs à des effets domino sont générés à cause des interdépen-

2. "The system of critical infrastructures [] constitutes the backbone of modern societies". 
dances. L'approche aléa-centrée ne suffit donc plus et doit par conséquent inévitablement s'orienter vers une approche système-centrée dans laquelle les conséquences de l'occurrence d'un aléa sur le système sont précisément étudiées : impacts directs sur les éléments du système situés dans la zone d'aléa, effets dominos parmi les autres éléments au sein du système, effets dominos parmi les autres systèmes connectés au système touché initialement. Dans ce contexte, l'émergence puis la mobilisation du concept de résilience depuis les années 2000, qu'il soit d'ailleurs vu comme une mutation, un bouleversement ou un simple glissement sémantique (Barroca et al., 2013) du concept de vulnérabilité, aborde pleinement cet aspect de territorialisation des risques en ce qu'elle place le système au centre de l'approche. Par l'utilisation de la résilience, donner ce rôle central aux systèmes sociotechniques urbains permet de les (re)placer comme actant ${ }^{3} \mathrm{~d}^{\prime}$ un territoire et leur donnant une grande importance dans la gestion des risques (Barroca, Serre, 2013). Par conséquent, la résilience est de fait territorialisée car elle accorde une place centrale à la singularité des lieux et participe de la reconnaissance de leurs capacités à faire face et à répondre en cas de survenue d'une catastrophe (Quenault, 2015). C'est la raison pour laquelle chaque tentative qui vise à appréhender l'état actuel et les progrès concernant la résilience d'un objet quel qu'il soit est inévitablement limitée si elle ne présente pas une base géographique solide ${ }^{4}$ (Weichselgartner, Kelman, 2014), d'autant que la question des emboîtements d'échelles spatiales est essentielle dans les études portant sur la résilience urbaine (Toubin, 2014). Néanmoins, la résilience est une notion dont la géographie des risques a recours depuis récemment à cause de limites aujourd'hui encore importantes liées à ce transfert tardif et qui ne va pas de soi depuis d'autres disciplines (Pigeon, 2012). Ainsi, les liens fondamentaux entre territoire et vulnérabilité perdurent et semblent s'orienter désormais vers des liens entre géographies et résilience. De même, l'approche jusqu'alors aléa-centrée dans l'analyse et la gestion des risques laisse ainsi place à une logique système-centrée mettant l'accent sur une vision élargie aux aspects systémiques et conséquences globale des risques.

\subsection{Gestion des risques et outils}

\subsubsection{Outils traditionnels de représentation territorialisée des risques}

L'outil traditionnel par excellence de la territorialisation des risques, de tous types, est l'outil cartographique. Dans le cas des risques naturels, la cartographie se décline en plusieurs types de cartographies selon les objets qui y sont représentés. Par exemple, une carte de vulnérabilité s'intéresse généralement à certaines composantes du système territorial comme la population, les établissements de santé ou le système d'approvisionnement en eau d'une ville (D'Ercole, Metzger, 2009) et expose leur vulnérabilité face à un aléa donné. Quel que soit le type de cartes utilisées pour

3. La notion d'actant a été exposée dans différents travaux de sociologie et est repris ici afin de signifier la volonté d'intégrer les acteurs (humains) et les systèmes techniques dans une même analyse.

4. "Attempts to capture the state of and progress towards resilience can therefore be limited without a solid geography foundation, showing how geographers can and should be involved in this work." 
la représentation des risques, leur but final reste identique : déterminer l'aménagement urbain qu'il est possible de réaliser sur un territoire au vu de sa propension à être touché par des aléas. Néanmoins, en termes d'usages, le reproche majeur qu'il est possible de faire sur l'outil cartographique appliqué aux risques est qu'il reste l'affaire des experts ou tout du moins d'acteurs spécifiques, dans sa traduction et son utilisation. C'est en ce sens que les outils plus récents de gestion territorialisée des risques apportent des réponses à ces nouveaux enjeux de représentation, de visualisation et de communication.

\subsubsection{Outils récents de gestion territorialisée des risques}

Comme ceci a pu être constaté dans des domaines aussi divers que ceux de la gestion des zones littorales ou du suivi et de l'analyse des risques côtiers (Gourmelon et al., 2006), les progrès technologiques réalisés au cours des deux dernières décennies dans le domaine des Technologies de l'Information Géographique (TIG) et de la Communication (TIC) ont fourni un contexte favorable au développement de la cartographie comme un outil de travail, de communication et d'aide à la décision car elle facilite l'observation et l'analyse des phénomènes sur un espace et dans le temps (Arnaud, 2015). Ces développements techniques et disciplinaires se regroupent aujourd'hui sous le terme de géomatique. En effet, la géomatique se définit comme une approche systémique, multidisciplinaire et intégrée dont le but est de concevoir des méthodes et modèles innovants notamment pour mieux rendre compte de la composante spatiale des phénomènes étudiés et aussi de sélectionner des outils et techniques autour de données géoréférencées spatialement ${ }^{5}$ (Gomarasca, 2009). Néanmoins, bien que les domaines applicatifs de la géomatique soient variés, elle reste une activité scientifique et de recherche dont le produit est pour certains auteurs le Système d'Information Géographique (SIG) (Joliveau, 2004). En effet, grâce à une certaine démocratisation des TIG qui a conduit de nombreux acteurs des territoires à s'approprier des outils issus de la géomatique (Noucher et al., 2008), le SIG est sans doute l'outil phare qui a su le mieux bénéficier de cette mouvance. Aujourd'hui, le SIG s'adresse aux acteurs du territoire, des concepteurs aux exploitants en passant par les usagers ou les habitants, qu'ils soient spécialistes ou non. Dans le contexte particulier des risques pour lequel l'aide à la décision est primordiale dans leur gestion et leur maîtrise, un certain nombre d'applications existent de SIG traitant les risques naturels affectant des systèmes techniques urbains. Le Système d'Information sur les Risques naturels de la haute Vallée de l'Arve (SIRVA) est un exemple de prototype de SIRS ayant pour objectif de fournir une assistance aux gestionnaires des risques en montagne en rassemblant et recensant les différentes informations et de mettre à disposition un outil fournissant de l'information préventive. Les risques étudiés sont les avalanches ou encore les mouvements de terrain. SIRVA permet aux gestionnaires

5. "Geomatics is defined as a systemic, multidisciplinary, integrated approach to selecting the instruments and the appropriate techniques for collecting, storing, integrating, modeling, analyzing, retrieving at will, transforming, displaying, and distributing spatially georeferenced data from different sources with welldefined accuracy characteristics and continuity in a digital format." 
de récupérer et sécuriser des données existantes, en intégrer de nouvelles au fur et à mesure de leur production et faciliter leur consultation (Manche, 2000; Manche et al., 2002). Un autre exemple d'utilisation de système d'information pour la gestion des risques est celui d'un Web SIRS pour l'évaluation de la résilience urbaine des réseaux techniques en vue d'un partage d'informations entre les gestionnaires des réseaux (Serre, 2015). Le risque naturel étudié dans ce Web SIRS est le risque inondation. L'outil a pour vocation d'intégrer des indicateurs d'aide à la décision basés sur trois capacités considérées comme primordiales pour étudier la résilience des réseaux techniques urbains. Ces indicateurs peuvent être calculés grâce au Web SIRS. La résilience y est alors évaluée sur un plan technico-fonctionnelle, et parallèlement, d'autres outils en cours de développement visent à l'évaluer d'un point de vue organisationnel (Toubin et al., 2013). De plus, (Fekete et al., 2015) synthétisent quelques contributions majeures dans l'utilisation de SIG pour la gouvernance des risques. Les auteurs démontrent que ces utilisations de SIG sont faites par une importante diversité d'acteurs tels que les collectivités publiques, les gestionnaires industriels de risques ou encore les organismes de secours et sauvetage. Ils montrent également l'émergence d'un grand nombre d'usages qu'il est possible de faire des SIG en rapport avec la gestion des risques, notamment lorsque les SIG sont couplés à des modèles de simulation : évaluation du temps d'évacuation de zones urbaines en cas de risque naturel ou de risque terroriste, évaluation d'indicateurs socio-économiques dans un contexte de changement climatique, d'indicateurs démographiques... Ces usages apparaissent donc tout à fait appropriés aux infrastructures critiques soumises à différents types de risques. Ainsi, les SIG sont alors vus comme un «couteau suisse» polyvalent pour analyser, collecter et agréger des informations relatives à la gestion des risques ${ }^{6}$. Ces exemples révèlent le rôle clef que peuvent jouer les SIG dans la gestion des risques afin de relier des données issues de différentes sources de collaboration et les analyser de manière intégrative et globale ${ }^{7}$ (Fekete et al., 2015).

\section{Caractérisation de la vulnérabilité des systèmes de transport guidé face au risque hydrologique}

\subsection{D’un modèle de fonctions vers un modèle de données}

\subsubsection{Analyse structurelle}

La complexité croissante des systèmes techniques urbains tels que les systèmes de transport guidé nécessite une réelle réflexion préalable à toute analyse de risque les affectant. Car, cette notion de système complexe, c'est-à-dire de système constitué d'un nombre important d'éléments qui interagissent entre eux de façon non linéaire (Simon, 1991), a pour conséquence de rendre la plupart des approches physiques classiques

6. "GIS is [...] a Swiss Army Knife version regarding its versatility in combining and analyzing information, gathering and aggregating it."

7. "In this context of risk management and governance, GIS [...] can play a pivotal role not just in delivering data but also in connecting and analyzing data in a more integrative, holistic way." 
peu voire non efficaces pour produire un modèle de fonctionnement (Zwingelstein, 1996; Villemeur, 1997) du système. Cette situation a offert un regain d'intérêt à un certain type de méthodes pour l'analyse des risques impactant les systèmes techniques urbains : les méthodes issues de la sûreté de fonctionnement. La sûreté de fonctionnement consiste en effet à connaître, évaluer, prévoir, mesurer et maîtriser les défaillances de systèmes technologiques, tout comme les défaillances humaines, afin de limiter les conséquences de ces défaillances sur la santé, la sécurité des personnes, la productivité et l'environnement (Noyes, Peres, 2007). Les méthodes de sûreté de fonctionnement se détachent d'une certaine façon de la complexité intrinsèque des systèmes techniques urbains en élaborant une modélisation fonctionnelle du système, base de l'analyse de risque. Plus exactement, cette modélisation consiste à déterminer les interactions entre les composants d'un système et son environnement de façon à établir de manière formelle les liens entre les défaillances des fonctions, leurs causes et leurs effets (Serre et al., 2007). L'analyse fonctionnelle est une des méthodes issues de la sûreté de fonctionnement les plus usuellement appliquées, seule ou préalablement à d'autres méthodes. Ainsi, depuis une quinzaine d'années, l'analyse fonctionnelle s'est vu appliquée à de nombreux systèmes complexes de type urbain - systèmes de transport routier, ferré, systèmes de gestion des déchets, systèmes et ouvrages de protection... - (Gonzva et al., 2015) offrant des résultats de recherche et opérationnels pour les gestionnaires, exploitants et concepteurs de systèmes techniques urbains. D'un point de vue méthodologique, l'analyse fonctionnelle est précédée d'une analyse dite structurelle qui permet de déterminer les composants du système et les éléments de son environnement. L'analyse structurelle a conduit à l'identification des éléments suivants (FIGURE 2) :

- Contrôle-commande et signalisation : permet de garantir la circulation des trains en toute sécurité ;

- Énergie : correspond à la fourniture en énergie de traction du matériel roulant ;

- Gare ;

- Infrastructure : correspond aux équipements fixes relatifs aux voies ;

- Matériel Roulant : correspond au train transportant les usagers;

- Poste de Commandement Centralisé (P.C.C.) : assure l'exploitation par une gestion en temps réel du système de transport;

- Site de Maintenance des Infrastructures (S.M.I.) : assure la maintenance des équipements fixes relatifs aux voies, à l'énergie et au contrôle-commande et signalisation;

- Site de Maintenance et de Remisage (S.M.R.) : assure la maintenance en atelier du matériel roulant et son remisage en période hors-exploitation. De plus, l'environnement du système se compose de :

- Usagers : utilisent le système de transport guidé pour leurs déplacements ;

- Sous-stations électriques : assurent l'alimentation en énergie électrique d'une section de ligne. 


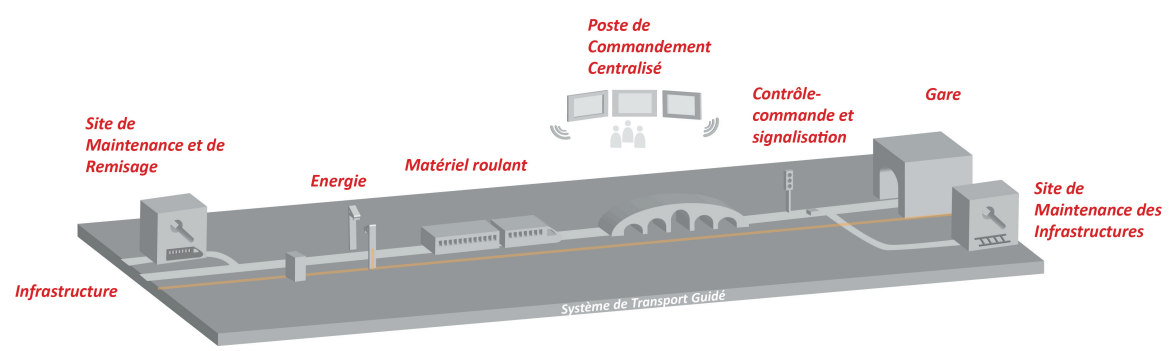

Figure 2. Analyse structurelle d'un système de transport guidé (Gonzva, Gautier, 2014)

\subsubsection{Analyse fonctionnelle : les Blocs-diagrammes Fonctionnels}

Suite à l'analyse structurelle, l'analyse fonctionnelle à proprement parler permet de déterminer les types d'interactions qu'entretiennent les composants du système. Puis, sous forme d'une schématisation précisément issue de la méthode APTE, le Bloc-diagramme Fonctionnel $(\mathrm{BdF})$ représentant le système est réalisé. Le BdF est une représentation graphique du système qui permet la mise en évidence des fonctions qui existent entre les composants du système et les éléments de son environnement extérieur, et entre les composants eux-mêmes du système. Les BdF d'un système de transport guidé ont été faits pour plusieurs conditions, d'une part selon la configuration d'implantation du système - en surface ou en souterrain - et d'autre part selon la situation du système - normale ou en crise, c'est-à-dire lorsque survient un aléa inondation. En situation de crise, le BdF permet de mettre en avant les relations de vulnérabilité entre l'aléa lui-même et les sous-systèmes, traduisant l'impact différent qu'a l'aléa sur les éléments du système (FIGURE 3).

\subsubsection{Analyse des modes de défaillances et de leurs effets}

Une autre méthode, usuellement appliquée consécutivement à l'analyse fonctionnelle, est l'Analyse des Modes de Défaillances et de leurs Effets (AMDE). C'est une méthode également issue de la sûreté de fonctionnement et qui est particulièrement efficace pour l'analyse des modes de défaillance en permettant de structurer, sous forme de tableaux, les informations sur les dégradations : les pertes de performance, leurs causes, leurs effets (Talon et al., 2009). En considérant un à un les composants du système et en analysant leurs modes de défaillance, cette méthode permet de mieux appréhender comment le système fonctionne et dysfonctionne avant, pendant et après un aléa.

D'autres modèles existent pour des travaux similaires d'analyse des défaillances des systèmes. Par exemple, l'Analyse Préliminaire des Dangers est utilisée dans les premières phases de conception d'un système et constitue un premier outil d'identification des risques potentiels (Villemeur, 1997). Cette méthode ne présente pas d'intérêt pour les systèmes techniques urbains tels que les systèmes de transport dans la mesure où les mécanismes de défaillance sont généralement bien identifiés. L'analyse du fonctionnement de ces systèmes relève d'études de sûreté plus fines telles que 


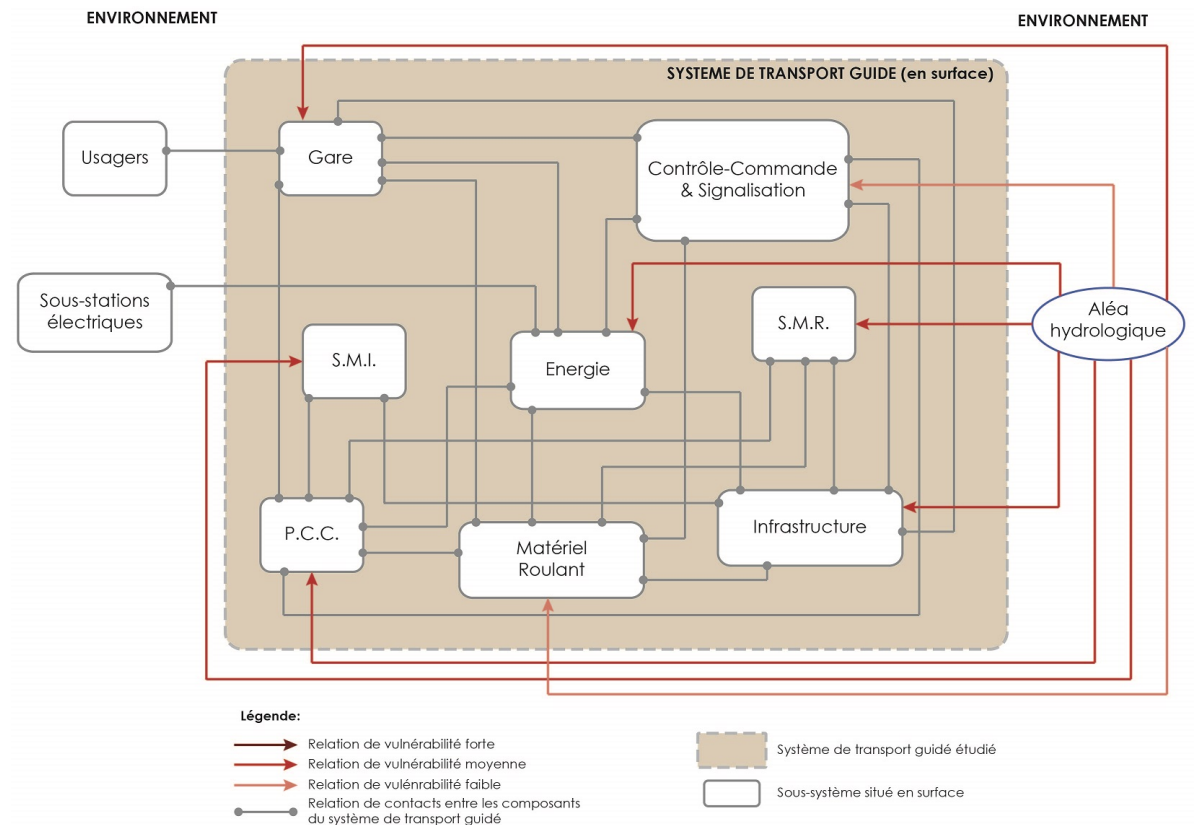

Figure 3. Bloc-diagramme Fonctionnel $(B d F)$ d'un système de transport guidé en situation de crise

l'AMDE (Serre, 2011). Autre exemple, les EBIOS, pour «Expression des Besoins et Identification des Objectifs de Sécurité », sont utilisées pour gérer les risques portant sur un secteur d'activités, un organisme dans son intégralité, une sous-partie ou des processus particuliers de celui-ci ou encore un système informatique (ANSSI, 2010). La méthode s'emploie également pour étudier un sujet global, tel qu'un système complexe composé de plusieurs sous-systèmes, pour ensuite se focaliser sur un sous-ensemble. Néanmoins, les EBIOS sont adaptés pour l'expression d'objectifs de sécurité et la détermination d'exigences de sécurité de l'objet étudié (DCSSI, 2004) tandis que les besoins méthodologiques de cette recherche abordent des objectifs de continuité fonctionnelle et des exigences de maintien de l'intégrité du système étudié dans un contexte de risques. L'AMDE semble donc plus adaptée à ces doubles exigences fonctionnelles et de conservation du système. La méthode HAZOP pour «HAZard and Operability studies » présente des similitudes avec l'AMDE. Cette méthode consiste, à partir de mots-clés, à passer en revue les déviations de paramètres importants du système ou du processus étudié en mettant en évidence les causes et conséquences de leurs déviations éventuelles ainsi que les moyens de détection et actions correctrices possibles (Zwingelstein, 1996). Or, la méthode HAZOP, contrairement à l'AMDE, n'est pas applicable lors de la phase de conception d'un projet d'ingénierie mais lors de la phase d'ingénierie de détail précédant la construction du système (Royer, 2009). Bien que la méthode HAZOP s'inscrive également dans la sûreté de fonctionnement, elle est centrée sur l'installation à la différence de l'AMDE qui est 
centrée sur les composants. Ainsi, une AMDE a été réalisée pour deux configurations d'implantation, lorsqu'ils subissent une perturbation de type aléa hydrologique, pour un système de surface et souterrain. Synthétisée sous forme de tableau, les résultats de l'AMDE listent : les composants un à un, avec un identifiant, le sous-système dans lequel il est inclus, les fonctions numérotées que ce composant assure, ses modes de défaillance qui correspondent à la non réalisation de ses fonctions suite à un aléa, les évènements qui ont causé la survenue de ces modes de défaillance et, enfin, les effets sur l'ensemble du système de la dysfonction de ce composant (FIGURE 4).

\begin{tabular}{|c|c|c|c|c|c|}
\hline Compo. & $\begin{array}{c}N^{\circ} \\
\text { Fonct. }\end{array}$ & Fonction & Défaillance & $\begin{array}{c}\text { Causes de la } \\
\text { défaillance de la } \\
\text { fonction }\end{array}$ & $\begin{array}{c}\text { Effets de la } \\
\text { défaillance de la } \\
\text { fonction }\end{array}$ \\
\hline \multirow{4}{*}{ Ballast } & 47 & $\begin{array}{l}\text { - Permettre la stabilité de } \\
\text { circulation du matériel } \\
\text { roulant }\end{array}$ & $\begin{array}{l}\text { - Ne permet pas la } \\
\text { stabilité de circulation } \\
\text { du matériel roulant }\end{array}$ & $\begin{array}{l}\text { - Dislocation de la voie } \\
\text { ferrée }\end{array}$ & $\begin{array}{l}\text { - Interruption de la } \\
\text { circulation ferroviaire }\end{array}$ \\
\hline & 48 & $\begin{array}{l}\text { - Répartir les charges sur } \\
\text { la plate-forme } \\
\text { - Immobiliser les traverses } \\
\text { dans leur position } \\
\text { - Assurer le drainage des } \\
\text { eaux zénithales }\end{array}$ & $\begin{array}{l}\text { - Ne répartit les charges } \\
\text { sur la plate-forme } \\
\text { - N'immobilise pas les } \\
\text { traverses dans leur } \\
\text { position } \\
\text { - N'assure pas le } \\
\text { drainage des eaux } \\
\text { zénithales }\end{array}$ & $\begin{array}{l}\text { - Emportement du } \\
\text { ballast } \\
\text { - Submersion du ballast }\end{array}$ & $\begin{array}{l}\text { - Dislocation de la } \\
\text { voie ferrée }\end{array}$ \\
\hline & 49 & $\begin{array}{l}\text { - Résister aux pressions } \\
\text { hydromécaniques } \\
\text { exercées par la crue } \\
\text { - Éviter la submersion } \\
\text { - Éviter l'emportement }\end{array}$ & $\begin{array}{l}\text { - Ne résiste pas aux } \\
\text { pressions } \\
\text { hydromécaniques } \\
\text { exercées par la crue } \\
\text { - N'évite pas la } \\
\text { submersion } \\
\text { - N'évite pas } \\
\text { l'emportement }\end{array}$ & $\begin{array}{l}\text { - Débit trop grand } \\
\text { - Hauteur d'eau trop } \\
\text { grande } \\
\text { - Duree d'immersion } \\
\text { trop grande } \\
\text { - Charriage d'éléments } \\
\text { massifs par la crue }\end{array}$ & $\begin{array}{l}\text { - Emportement du } \\
\text { ballast } \\
\text { - Submersion du } \\
\text { ballast }\end{array}$ \\
\hline & 50 & $\begin{array}{l}\text { - Assurer le maintien } \\
\text { physique de la plate- } \\
\text { forme voie durant la crue }\end{array}$ & $\begin{array}{l}\text { - N'assure pas le } \\
\text { maintien physique de la } \\
\text { plate-forme voie durant } \\
\text { la crue }\end{array}$ & $\begin{array}{l}\text { - Hauteur d'eau trop } \\
\text { grande } \\
\text { - Charriage d'éléments } \\
\text { massifs par la crue }\end{array}$ & $\begin{array}{l}\text { - Emportement du } \\
\text { ballast } \\
\text { - Dislocation de la } \\
\text { voie ferree }\end{array}$ \\
\hline
\end{tabular}

Figure 4. Extrait de l'AMDE pour le composant «ballast » d'un système de transport guidé en surface (Gonzva et al., 2014)

\subsubsection{Les graphes causaux}

Enfin, une troisième méthode permet de représenter les résultats obtenus lors de l'AMDE : les graphes causaux. Ces graphes d'évènements causaux, proches des arbres de défaillance, des causes et d'événement (Zwingelstein, 1996), représentent graphiquement une séquence d'évènements constituée d'un évènement initiateur et d'une combinaison de défaillances successives. D'autres méthodes existent pour des travaux similaires de représentation de défaillances des systèmes. Les réseaux bayésiens, par exemple, sont des modèles graphiques représentant des relations causales mais dont le but est de quantifier ces relations qui existent entre des variables aléatoires. Or, dans cette recherche, le but est ici de représenter l'existence de relations causales de défaillances de façon qualitative donc, sans nécessairement avoir de considérations quantitatives de l'ensemble. Ces graphes mettent en évidence les relations de causes et d'effets qui existent entre les défaillances. En effet, les dysfonctionnements des composants se diffusent de proche en proche (cause initiale générant un effet 1, générant lui-même un effet 2 , générant un effet $i$...) créant des scénarios de défaillances représentés par des graphes causaux (FIGURE 5). Dès lors que, pour chacun des com- 
posants, il n'existe pas de nouvel effet (itération $n+1$ ) à associer à une cause (itération $n$ ), le graphe causal s'arrête. Ces scénarios reflètent donc l'interdépendance fonctionnelle qui existe entre l'ensemble des composants du système et c'est cette interdépendance inhérente aux éléments qui conduit à des défaillances en chaîne et, finalement, au dysfonctionnement général du système de transport.

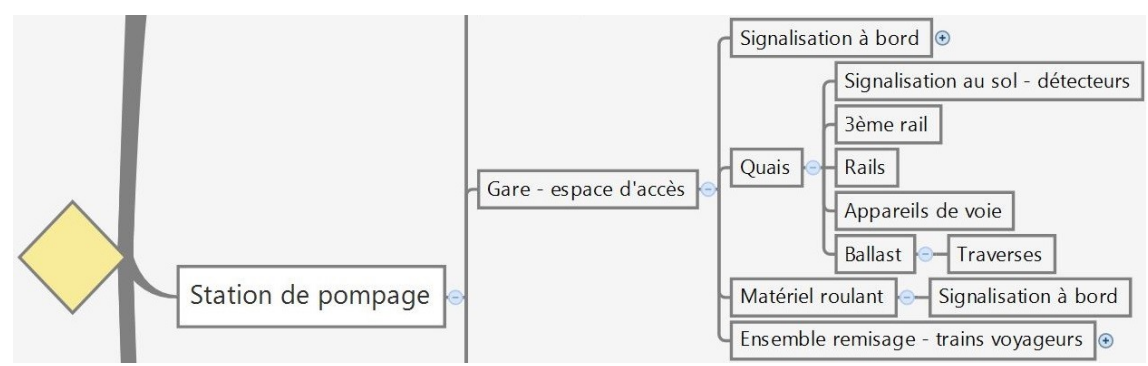

Figure 5. Extrait d'un scénario de défaillances successives de composants représenté sous forme de graphe causal

\subsubsection{Transposition des BdF vers des modèles de données relationnels géographiques}

Les modélisations de systèmes, quels qu'ils soient, peuvent se faire de plusieurs façons. Par ailleurs à la modélisation fonctionnelle présentée précédemment existent d'autres types de modélisations que sont les modèles relationnels de données, en particulier ceux appliqués au domaine de la géographie. De façon conceptuelle, un modèle relationnel de données consiste en une manière de modéliser et d'ordonner les relations qui existent entre plusieurs données ou plusieurs informations. Appliqué au domaine de la géographie, ces principes conceptuels ont donné lieu aux bases de données géographiques, outils opérationnels qui permettent d'organiser et de gérer l'information géographique sous forme numérique. Ce sont sur ces bases de données relationnelles géographiques, ou géodatabases, que reposent les SIG. Les SIG interrogent, en effet, les géodatabases sur les données qu'elles contiennent afin d'en extraire des informations.

Le modèle relationnel proposé dans la suite et développé à partir d'une modélisation fonctionnelle et dysfonctionnelle (c'est-à-dire lorsque le système étudié fait face à un aléa pouvant générer des risques de dysfonctionnements) peut aboutir à la construction d'une ontologie spécifique. En effet, bien que les bases de données relationnelles demeurent un des moyens les plus répandus pour stocker, rechercher et manipuler des données, la structure et les contraintes d'intégrité du modèle relationnel sont définies par des schémas qui ne sont pas aussi expressifs que des ontologies pour ce qui est de la représentation de la sémantique des données. Il s'avère donc essentiel de construire des ontologies qui soutiennent sémantiquement l'information contenue dans ces modèles de données relationnelles (Benslimane et al., 2006). Des logiciels permettent alors, une fois le modèle relationnel élaboré et la base de données associée, d'en dériver une ontologie précise et instanciée (Cerbah, 2008) ou alors des ap- 
proches de type rétro-ingénierie peuvent également permettre la construction d'ontologies (Benslimane et al., 2006).

Les deux types de modélisations précédemment présentées, bien qu'elles soient issues de domaines techniques et scientifiques tout à fait différents et donc appliquées usuellement à des systèmes très distincts, offrent une possible transposition d'ordre méthodologique de l'une vers l'autre. Cette transposition, ou tout du moins la possibilité d'utiliser une représentation pour construire l'autre, semble en effet possible du modèle fonctionnel, représenté par le BdF, vers le modèle de données relationnel et réciproquement à partir d'un parallèle entre les éléments constitutifs de chacun des modèles. Ce passage paraît intuitivement possible dans la mesure où les systèmes de transport guidé, et plus généralement les systèmes techniques urbains, sont des objets géographiques avec une position spatiale définie au sein d'une ville. Plus précisément, ce passage entre ces deux modèles est tout d'abord permis grâce à la structuration commune des éléments des modèles. En effet, dans un modèle fonctionnel, il existe une hiérarchie entre les éléments selon le niveau de précision souhaitée pour le modèle ; le modèle représente alors un système, constitué de sous-systèmes, euxmêmes dissociables en composants, puis en sous-composants, voire en éléments plus fins selon les besoins. De façon similaire, un modèle de données respecte une classification entre ses éléments ; le modèle représente alors un système, constitué de tables, elles-mêmes composées de champs pour lesquels des attributs sont définis. De plus, ce passage entre ces deux modélisations est permis par l'idée commune d'établir des liens entre les éléments des modèles. En effet, dans un modèle fonctionnel existent entre les éléments des interactions qui sont représentatives des fonctions assurées par le système; dans un modèle de données, des relations sont établies entre les tables afin de mettre en évidence les liens qui existent entre celles-ci. Dans le cas du modèle de données, on peut faire appel au modèle ou diagramme entité-association pour décrire graphiquement un tel modèle conceptuel; les entités sont les objets qui constituent le modèle et les associations représentent les liens entre objets. C'est, en règle générale, à partir des modèles objet et entité-association que se conçoivent les bases de données. Dès lors, il est ainsi possible d'associer deux à deux les éléments qui constituent chacun des modèles comme le montre le TABLEAU 1.

Tableau 1. Transposition entre un modèle de données géographiques relationnel et un modèle fonctionnel

\begin{tabular}{ccc} 
modèle relationnel & & modèle fonctionnel \\
\hline table & $\leftrightarrow$ & sous - système \\
champ & $\leftrightarrow$ & composant \\
enregistrement & $\leftrightarrow$ & sous - composant \\
attribut $_{\text {relation }}^{*}$ & $\leftrightarrow$ & - \\
retion
\end{tabular}

* La notion de relation utilisée ici se fait au sens d'une liaison ou association; elle ne doit pas être confondue avec la notion de relation utilisée dans l'algèbre relationnelle, qui correspond à une table. On utilisera par la suite uniquement cette première définition pour la notion de relation. 
Il est important de noter que l'élément «attribut » du modèle relationnel ne possède a priori pas d'équivalent au sein du modèle fonctionnel, du moins pas de façon aussi évidente que les autres éléments. Nous montrons dans la suite que l'information géographique vient combler ce manque ontologique pour rendre finalement la transposition d'un modèle à l'autre complète et théoriquement possible.

\subsection{Construction d'un modèle de données relationnel géographique relatif à un système de transport guidé}

La partie suivante décrit d'un point de vue pratique les étapes de transposition d'un modèle fonctionnel de système de transport guidé vers un modèle relationnel de système de transport guidé. En effet, bien que validé théoriquement sur le principe dans le paragraphe précédent, le passage d'un modèle à l'autre nécessite plusieurs étapes de construction. Le point de départ de la transposition se base sur les résultats de l'analyse fonctionnelle, à savoir les Blocs-diagrammes Fonctionnels qui mettent en évidence les sous-systèmes et leurs fonctions. L'intérêt de cette recherche se concentrant sur la gestion des risques et l'apport de l'information géographique, les étapes suivantes se font sur le modèle du système lorsqu'il est en situation de crise, c'est-à-dire lorsqu'il fait face à un aléa inondation.

\subsubsection{Etape 1 : détermination des éléments d'un modèle à l'autre}

Cette étape consiste en fait à déterminer les objets du modèle entité-association, c'est-à-dire la partie «entité » (FIGURE 6). Elle consiste alors à transposer les soussystèmes en tables (TABLEAU 1).

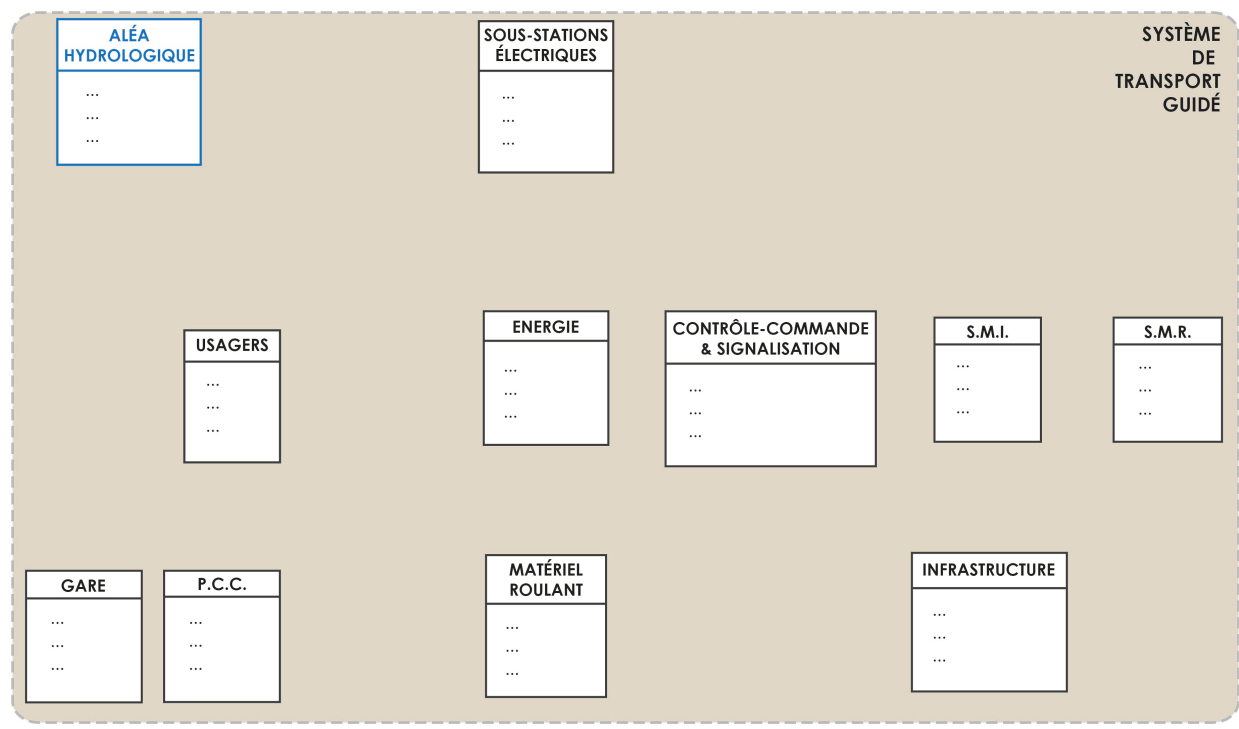

Figure 6. Première étape de la transposition - détermination des entités 


\subsubsection{Etape 2 : association des éléments d'un modèle à l'autre}

L'étape 2 consiste à déterminer les relations entre les objets du modèle. Elle consiste alors à transposer les fonctions en relations (TABLEAU 1). Par exemple, le modèle fonctionnel a permis d'identifier la fonction suivante : l'infrastructure assure le support du matériel. C'est la raison pour laquelle une association «supporter » relie les tables «matériel roulant» et «infrastructure».

On obtient ainsi le diagramme entité-association du modèle (FIGURE 7).

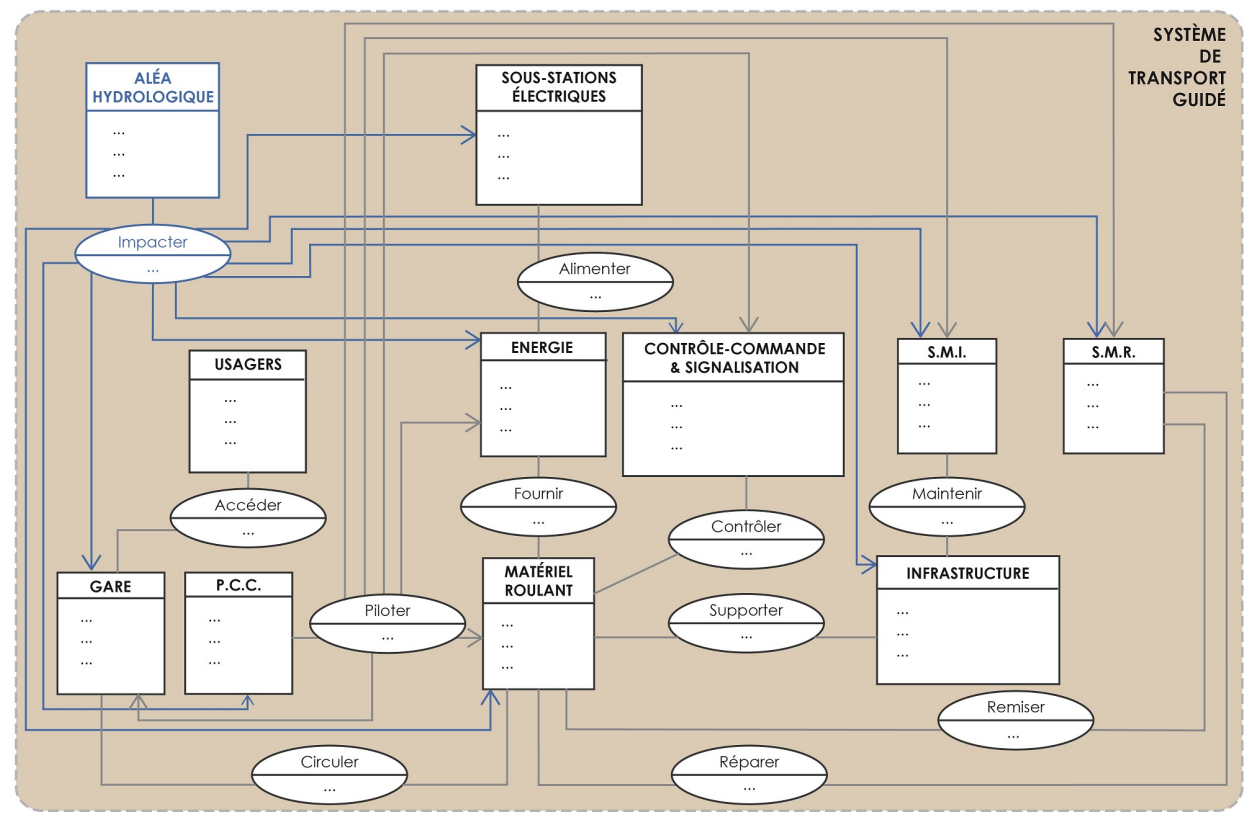

Figure 7. Deuxième étape de la transposition - détermination des associations

\subsubsection{Etape 3 : ajout des attributs et cardinalités au sein des champs}

C'est à cette étape qu'est mobilisée l'information géographique. Elle joue le rôle d'attributs dans le modèle relationnel et permet l'intégration de caractéristiques géographiques ; en quelque sorte, l'information géographique permet de spatialiser le modèle fonctionnel afin d'obtenir un modèle relationnel géographique complet du système de transport guidé.

Les attributs, pour chaque table du modèle de données, correspondent à :

- Un niveau géométrique : les champs représentent des objets surfaciques (cas d'une gare, d'une sous-station électrique par exemple), linéaires (cas de l'infrastructure par exemple) ou ponctuels (cas du matériel roulant par exemple);

- Un niveau sémantique : chaque champ possède, d'une part, des attributs spatiaux $(x ; y ; z)$ qui permettent de localiser un sous-système et d'autre part, des attributs temporels $\left(t_{1} ; D_{t} ; t_{2}\right)$ qui traduisent respectivement l'instant où survient l'inondation, sa durée et sa fin. 
Le niveau sémantique peut être fortement enrichi afin que l'apport de l'information géographique et temporelle ne se résume pas qu'à une donnée de hauteur, de largeur de composant ferroviaire. Des pictogrammes spatiaux et temporels peuvent, par exemple, rendre le modèle bien plus riche dans sa prise en compte d'information géographie et temporelle. Ces pictogrammes PVL, pour «Plug-in for Visual Language », correspondent à un langage graphique qui représente les propriétés géométriques, temporelles et visuelles des objets et des attributs étudiés ${ }^{8}$ (Brodeur et al., 2000). Les PVL sont alors stéréotypés et s'intègrent dans un outil CASE, pour «Computer-Assistant Software Engineering », appelé Perceptory qui repose sur des règle de modélisation précises. Par exemple, les caractéristiques spatiales d'une classe d'objets sont représentées à la gauche du nom de la classe et les caractéristiques temporelles sont à sa droite. La définition de caractéristiques spatiales et temporelles au niveau des attributs est également possible. Les pictogrammes spatiaux sont utilisés pour représenter les propriétés spatiales des concepts (forme ponctuelle, linéaire et polygonale, éléments 2D, 3D) et les pictogrammes temporels sont utilisés pour représenter les propriétés temporelles (existence ponctuelle ou existence durable) (Bédard, Larrivée, 2007) (FIGURE 8). Ces pictogrammes ont déjà été utilisés pour la modélisation, l'analyse et la gestion de réseaux de transports urbains afin de promouvoir l'utilisation de SIG dans ce cadre (S. Chen et al., 2008).

\begin{tabular}{|c|c|c|}
\hline & 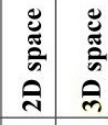 & Examples of cases \\
\hline $0 \mathrm{D}$ geometry & \begin{tabular}{|l|l|l}
$\bullet$ & 0 \\
\end{tabular} & hydrants when they are all represented by points \\
\hline \multirow{2}{*}{ 1D geometry } & 团 & road segments when they are all represented by lines \\
\hline & (0) & electric poles when they are all represented by vertical lines \\
\hline \multirow{2}{*}{ 2D geometry } & 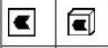 & lakes when they are all represented by polygons \\
\hline & 国 & walls when they are all represented by vertical plans \\
\hline 3D geometry & 國 & buildings when they are all represented by solids \\
\hline OD temporality & 0 & Existence of accidents; traffic flow of a road segment \\
\hline 1D temporality & Q & $\begin{array}{l}\text { Existence of a building; duration of its commercial use; } \\
\text { duration of its ownership by a given person }\end{array}$ \\
\hline
\end{tabular}

Figure 8. Pictogrammes spatiaux 2D et 3D et pictogrammes temporels (Bédard, Larrivée, 2007)

De plus, les associations peuvent également présenter des attributs d'ordre sémantique. Ces attributs caractérisent alors une ou plusieurs valeurs qui décrivent cette association et qui sont chacune catégorisée au sein d'un attribut. Par exemple, dans le cas de l'association «alimenter» entre les entités «gare» et «matériel roulant»,

8. "PVL (Plug-in for Visual Languages) [...] is a simple but powerful graphical notation depicting geometric, temporal and visual properties of objects and attributes". 
elle pourrait présenter un attribut «tension » prenant les valeurs « 750 volts », « 15000 volts » ou encore « 25000 volts » et un attribut « fréquence » prenant les valeurs « 50 hertz $»$ ou encore « 16,7 hertz ».

C'est à cette étape également que sont déterminées les cardinalités entre entités et associations. Les cardinalités correspondent au nombre minimum et maximum de fois où une occurrence d'entité participe dans des occurrences d'une association avec d'autres occurrences d'une ou plusieurs autres entités. Par exemple, dans le cas de l'association «piloter» qui relie les entités «P.C.C. » et «matériel roulant », les cardinalités sont les suivantes : un P.C.C. pilote au minimum un matériel roulant d'une ligne de transport et peut également en piloter sur plusieurs lignes de transport simultanément, d'où une cardinalité $(1, n)$; un matériel roulant relatif à une ligne de transport est nécessairement piloté par un P.C.C. et uniquement par un P.C.C. en charge de cette ligne, d'où une cardinalité $(1,1)$.

Le travail d'ajout des PVL au niveau des tables, des attributs et des cardinalités au sein des champs est présenté dans le modèle de données en FIGURE 9.

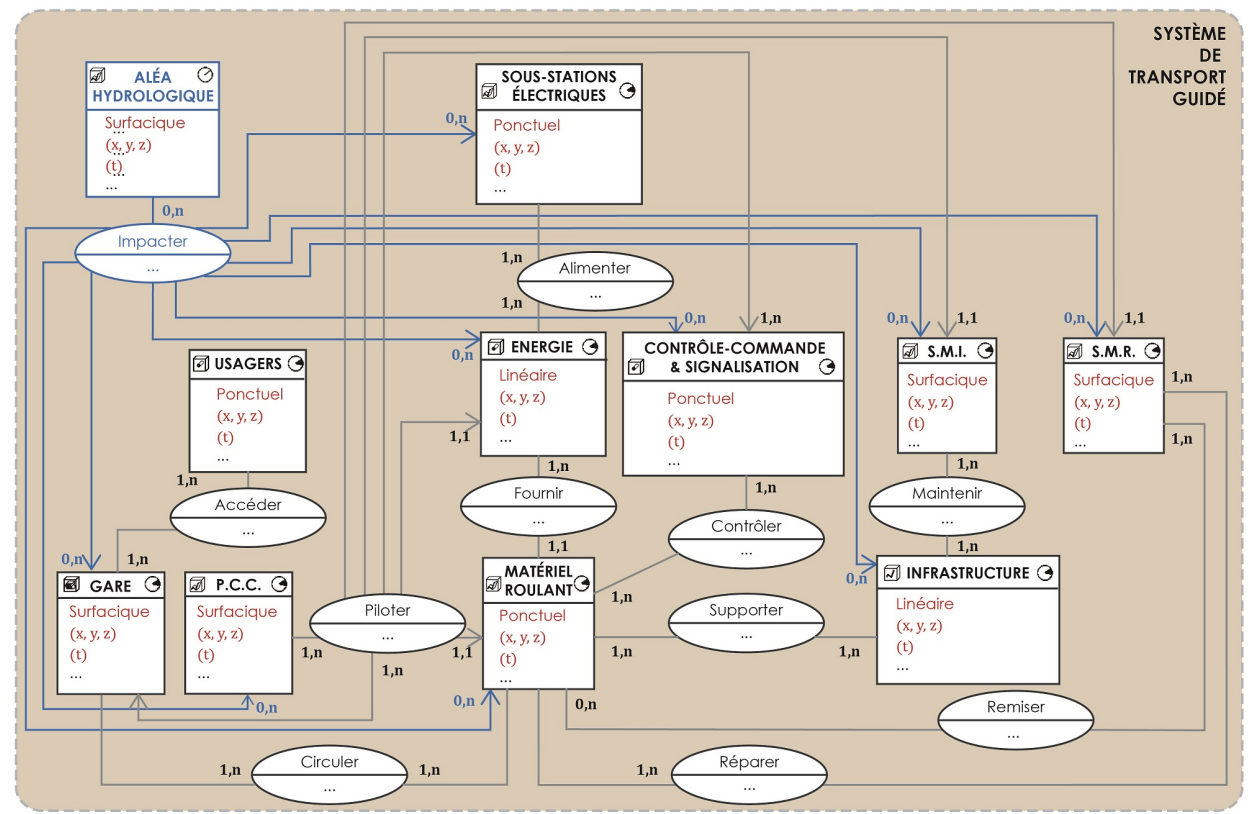

Figure 9. Troisième étape de la transposition - détermination des attributs et cardinalités

Par ailleurs, une façon d'enrichir la modélisation consiste à considérer des niveaux de granularité différents. Chaque objet peut alors être représenté de manière différente. Par exemple, la voie ferrée, en tant qu'objet de l'infrastructure, peut être géométriquement symbolisée par une ligne médiane avec un point de départ et un point d'arrivée ou peut être représentée par une agrégation de points la délimitant. Dès lors, selon la granularité choisie dans la représentation des objets, l'utilisation du modèle 
peut permettre de répondre à des interrogations plus ou moins précises : par exemple, l'identification simple des éléments du système impactés par l'aléa hydrologique ou la caractérisation précise de la surface d'impact de chaque élément par l'aléa.

\subsubsection{Etape 4 : élaboration d'une algèbre relationnelle}

La dernière étape consiste à déterminer les opérations élémentaires qu'il est possible de faire au sein du modèle relationnel dans le but d'interroger les données et d'en extraire des informations, dans notre cas utile pour la gestion des risques affectant les systèmes techniques urbains. L'objectif pratique de l'algèbre relationnelle consiste premièrement ici à croiser l'entité « aléa inondation » avec toutes les autres afin qu'il soit possible de déterminer les effets de l'aléa sur les composants du système.

Dans les faits, les relations liant l'entité «aléa hydrologique» aux autres entités ont un statut particulier. En effet, ces relations ne sont pas stricto sensu des associations, puisque ces relations ne sont pas permanentes, mais sont conditionnées par les caractéristiques géographiques de l'aléa et des autres entités. Par exemple, il est possible d'imaginer qu'un aléa n'impacte directement aucune gare si aucune gare ne se situe au sein de l'emprise géographique de l'aléa.

A partir de ces caractéristiques géographiques, une réflexion profonde doit ensuite être menée afin de préciser les attributs pouvant être associés à ces relations particulières. En l'occurrence, il semble nécessaire de caractériser les relations « impacter » par un attribut «niveau impact». Ce niveau d'impact peut alors être obtenu en croisant les caractéristiques de l'aléa (hauteur d'eau, vélocité...) et les caractéristiques des objets concernés (état de l'objet, dispositif de protection...). L'ensemble de ces réflexions mènera à l'élaboration de règles multicritères plus ou moins complexe (Malczewski, 2004 ; Y. Chen et al., 2010; Feizizadeh et al., 2014) et propre à chaque relation entre l'entité «aléa hydrologique » et les autres entités. Des premiers exemples simples de ce que peut produire une algèbre relationnelle comme informations nouvelles sont donnés ci-après dans le cas virtuel d'application.

\section{Mise en œuvre au sein d'un SIG du modèle de vulnérabilité : principes autour d'un cas d'application virtuel}

\subsection{Principes d'implémentation du modèle de données relationnel géographique}

Cette dernière partie s'intéresse à la mise en œuvre pratique du modèle de données relationnel géographique décrit précédemment de façon théorique au sein d'un SIG. La partie précédente a abouti au schéma entité-association permettant de concevoir la base de données à, ensuite implanter dans un SIG. La partie ci-dessous présente les typologies de données contenues dans cette base de données ainsi que les principes de l'algorithme de rétroaction qui contient les opérations à réaliser parmi ces données afin d'obtenir les défaillances indirectes, par effet domino, des composants du système. L'objectif est alors de déterminer les principes d'implémentation de ce modèle à partir d'un cas d'application virtuel simple de système de transport subissant un risque d'origine hydrologique. Différents niveaux d'information sont mis en évidence 
pour définir ces principes d'implémentation dans un SIG (FIGURE 10). Ils permettent de montrer les mécanismes complexes de vulnérabilité du système de transport en déclinant cette vulnérabilité selon trois niveaux : un niveau matériel, structurel et fonctionnel (Gleyze, 2005).

\subsubsection{Niveau matériel}

Cette première couche d'information constitue la base physique du système qui est étudié. En effet, le niveau matériel est défini par l'ensemble des sous-systèmes, composants, sous-composants et autres éléments qui constituent le système technique urbain étudié. Ce sont donc les éléments matériels du système de transport guidé, positionnés spatialement et temporellement, et qui sont susceptibles d'être soumis à un aléa hydrologique.

Les informations contenues dans ce niveau matériel sont issues du travail réalisé dans le cadre de l'analyse structurelle appliquée à un système de transport guidé.

\subsubsection{Niveau structurel}

Cette deuxième couche d'information aborde la notion de réseau existant entre les éléments matériels du système. En effet, lorsqu'un élément matériel est touché par un aléa, l'organisation du système est modifiée c'est-à-dire que les liaisons fonctionnelles entre cet élément et les autres ne sont plus toutes assurées. La structure réticulaire qui existe au sein du système en situation normale de fonctionnement est donc altérée suite à cet endommagement matériel initial. Ainsi, le niveau structurel prend en compte les interdépendances entre les éléments matériels.

Les informations contenues dans ce niveau matériel sont issues du travail réalisé dans le cadre de l'analyse fonctionnelle appliquée à un système de transport guidé.

\subsubsection{Niveau fonctionnel}

Ce troisième niveau d'information caractérise les effets sur le fonctionnement $\mathrm{du}$ système de défaillances d'éléments matériels. Une défaillance matérielle se traduit alors par une exploitation dégradée du système qui ne peut plus assurer un niveau nominal de service. Dans le cas d'un système de transport, l'exploitation en fonctionnement en mode dégradé peut, par exemple, se traduire par des vitesses de trains réduites ou des gares non desservies.

\subsubsection{Mécanisme de rétroaction}

Cependant, le travail d'analyse des modes de défaillances d'un système de transport guidé a montré que cet effet en cascade peut générer non pas une mais plusieurs séries de défaillances successives. Autrement dit, sur la base de la structure du système, un dysfonctionnement matériel initial peut engendrer la dysfonction d'autres éléments qui eux-mêmes peuvent être la cause de la défaillance d'une troisième série d'éléments matériels, et cela de proche en proche jusqu'à des endommagements matériels finaux. Ainsi, pour que les informations contenues dans le niveau fonctionnel soient exhaustives, il est nécessaire de mettre en place un mécanisme de rétroaction 
qui, pour chaque élément matériel identifié comme défaillant, réinterroge le niveau structurel pour déterminer les éléments matériels qui dépendent de celui-ci. De façon itérative, il est possible d'obtenir toutes les chaînes de défaillances successives et alors de caractériser l'impact général d'un aléa hydrologique sur l'ensemble du système. Finalement, les informations contenues dans ce niveau fonctionnel sont issues du travail réalisé dans le cadre de l'AMDE et des graphes causaux appliqués à un système de transport guidé.

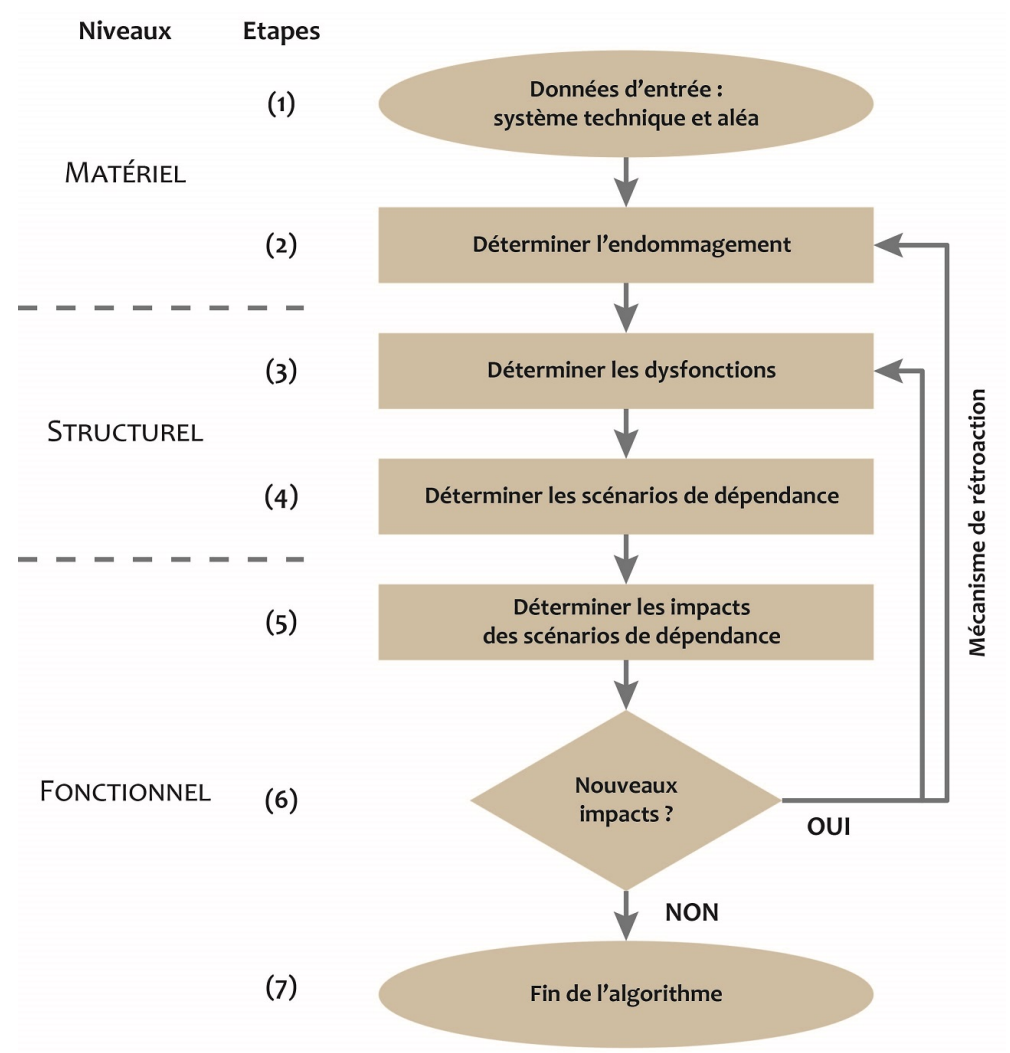

Figure 10. Algorithme d'implémentation du modèle de données au sein d'un SIG

\subsection{Application à un système de transport guidé virtuel}

Pour illustrer les principes de mise en œuvre pratique d'un modèle de données relationnel géographique au sein d'un SIG, un cas virtuel de système de transport est considéré. Les niveaux matériel et structurel définissent l'architecture du système considéré et les relations de dépendances et d'interdépendances au sein des éléments de cette architecture. Chaque élément possède des attributs spatiaux, c'est-à-dire que sa position spatiale est définie par des coordonnées $(x ; y ; z)$. Ces coordonnées représentent le centre de l'élément considéré. L'architecture est volontairement simplifiée 
et ne recense que quelques éléments très représentatifs d'un système de transport (FIGURE 11).

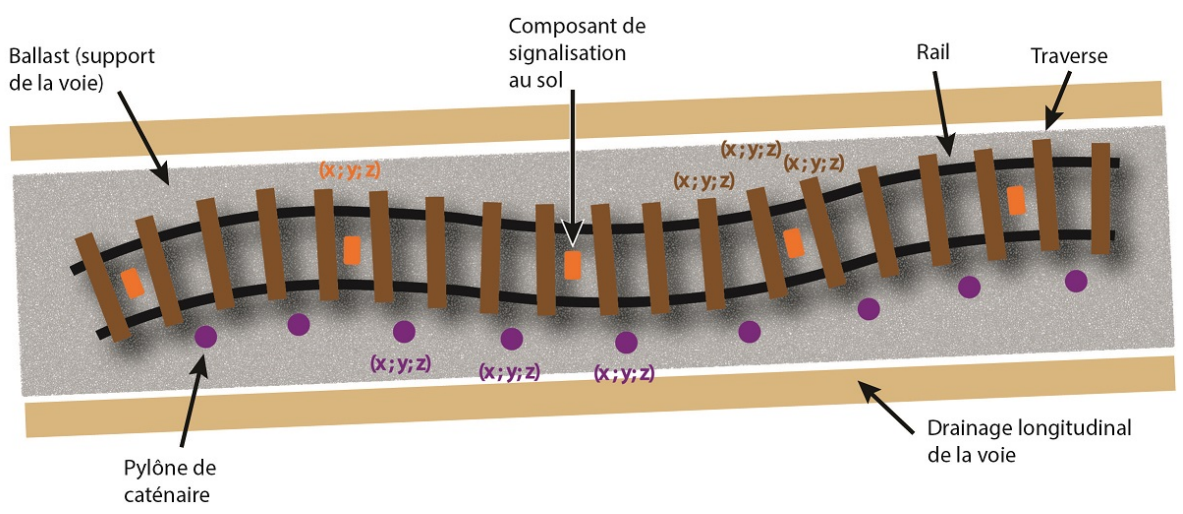

Figure 11. Description matérielle et structurelle du cas d'application virtuel

A partir du système ainsi défini, un aléa hydrologique vient impacter une section. La zone d'aléa est déterminée sous forme de patatoïde dont l'ensemble des points qui circonscrivent la surface sont spécifiés à partir de leurs coordonnées $(x ; y ; z)$ connus. Les éléments du système inclus dans la zone d'aléa sont directement impactés et deviennent donc défaillants. Cela correspond à des défaillances de premier ordre (FIGURE 12).

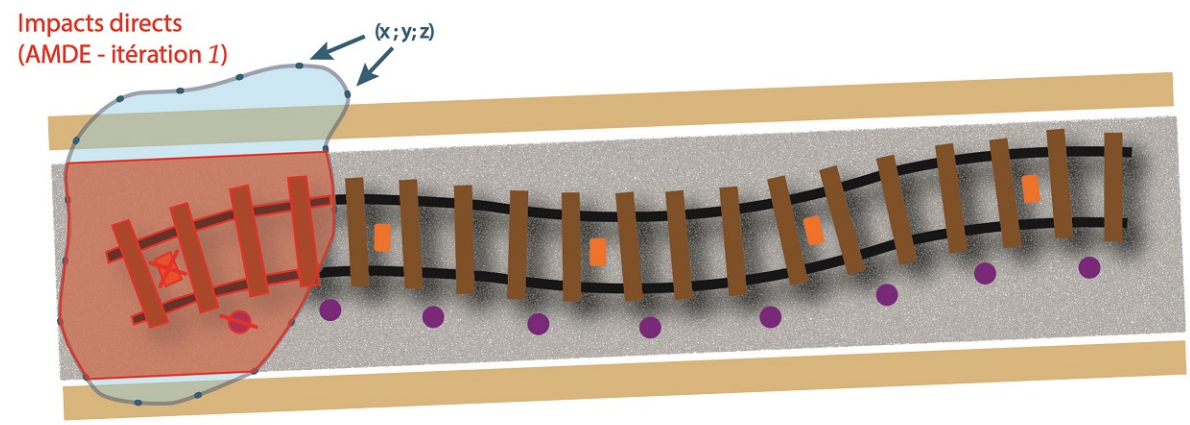

Figure 12. Impacts directs de l'aléa hydrologique sur le cas d'application virtuel

Puis, grâce à l'algorithme de rétroaction mis en place, l'AMDE est interrogée afin d'identifier si les composants défaillants par impact direct entraînent la défaillance d'autres composants par impact indirect ou effet domino. Cela correspond à des défaillances de deuxième ordre ou plus (FIGURE 13).

L'algèbre relationnelle établie permet des opérations élémentaires au sein du modèle dans le but d'interroger les données qu'il contient. Par exemple, dans une première étape, l'utilisateur peut entrer un degré d'intensité de l'inondation, de 1 à 5 , 


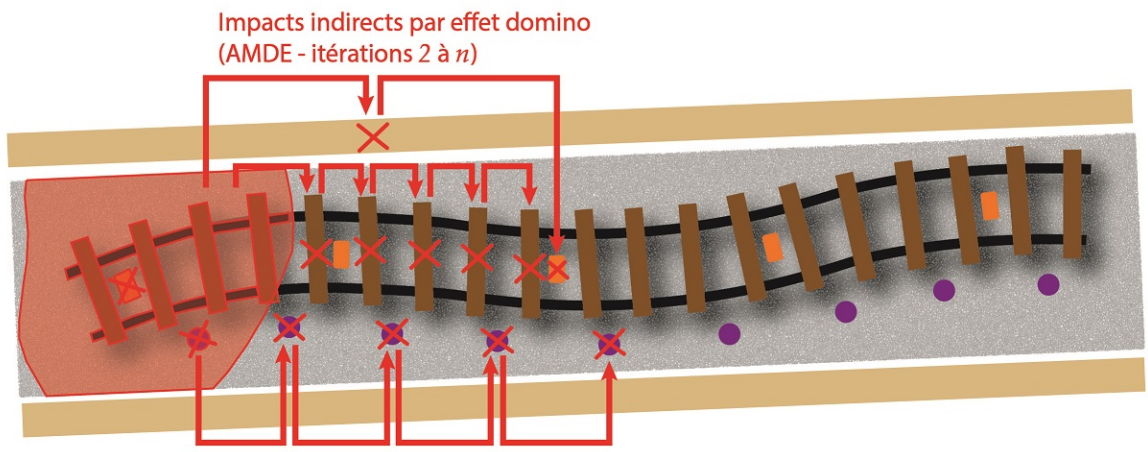

Figure 13. Impacts par effet domino de l'aléa hydrologique sur le cas d'application virtuel

selon que l'on soit dans le cas d'un évènement pluvial à faible période de retour ou d'une crue majeure. Puis, les composants se voient attribuer une valeur relative à leur état après avoir été touché par l'aléa, de 1 à 5 , selon qu'il ait été faiblement ou très fortement touché. Pour un composant donné $k$ ces deux valeurs seraient alors croisées selon des règles établies permettant de déterminer si, selon la valeur d'intensité de l'aléa et la valeur de l'état du composant, celui-ci fonctionne ou ne fonctionne plus. Dans une seconde étape, grâce au contenu de l'AMDE, il peut alors être possible de déterminer les autres composants $i j, m$ dont le bon fonctionnement dépend directement du bon fonctionnement du composant $\mathrm{k}$. L'impact global, direct et par effet domino, d'un risque hydrologique peut donc être de cette façon évalué quantitativement et spatialement (TABLEAU 2).

Tableau 2. Application des principes de l'algèbre relationnelle sur le cas d'application virtuel

Etape 1 : impacts directs

Etape 2 : impacts par effet domino

\begin{tabular}{cccccc}
\hline $\begin{array}{c}\text { aléa } \\
\text { hydrolo- } \\
\text { gique }\end{array}$ & $\mathbf{X}$ & $\begin{array}{c}\text { état du } \\
\text { compo- } \\
\text { sant }\end{array}$ & $\begin{array}{c}\text { fonction- } \\
\text { nement du } \\
\text { composant } k\end{array}$ & $\begin{array}{c}\text { AMDE } \\
\text { (dépendances } \\
\text { au composant }\end{array}$ & $\rightarrow \begin{array}{c}\text { fonction- } \\
\text { nement des } \\
\text { composants } i,\end{array}$ \\
$\mathbf{1}$ & $\mathbf{1}$ & Actif & composant $\boldsymbol{i}$ & $j, m$ \\
$\mathbf{2}$ & $\mathbf{2}$ & Hors-service & & $\begin{array}{c}\text { Actif } \\
\text { Hors-service }\end{array}$ \\
$\mathbf{3}$ & $\mathbf{3}$ & & composant $\boldsymbol{j}$ & $\begin{array}{c}\text { Actif } \\
\mathbf{4}\end{array}$ \\
$\mathbf{5}$ & $\mathbf{4}$ & & & Hors-service \\
& $\mathbf{5}$ & & composant $\boldsymbol{m}$ & Actif \\
& & & & & Hors-service
\end{tabular}




\section{Conclusions et perspectives de recherche}

Le développement de méthodologies et de technologies d'analyse des risques qui affectent les systèmes techniques urbains apparaît indispensable pour améliorer la résilience urbaine. Ces développements doivent, de plus, être transversaux en mobilisant plusieurs types de méthodologies et technologies dans le but d'être toujours plus performants, innovants et de couvrir un champ d'action urbain toujours plus large. Par ces développements, la gestion des risques est donc considérée comme un point d'entrée opérationnel pour l'amélioration de la résilience urbaine.

En appliquant ce travail à un système technique urbain particulier, les systèmes de transport public guidé, faisant face à un aléa donné, les inondations, l'innovation méthodologique présentée dans cet article a consisté à croiser des méthodes issues de deux domaines scientifiques, la sûreté de fonctionnement et la géomatique, pour l'élaboration d'outil méthodologique d'évaluation de la vulnérabilité. Les méthodes utilisées sont respectivement l'analyse fonctionnelle et l'analyse des modes de défaillances et de leurs effets d'une part, qui sont croisées avec les bases de données géographiques et systèmes d'information géographique d'autre part.

$\mathrm{Ce}$ croisement est motivé pour plusieurs raisons. Le premier argument est d'ordre contextuel car les systèmes techniques urbains tels que les systèmes de transport guidé sont avant tout des objets géographiques dans la mesure où ils sont spécifiquement implantés sur un territoire donné avec une localisation précise. Dès lors, la gestion des risques affectant les systèmes techniques urbains est de fait territorialisée, réflexion soutenue par une grande partie de la communauté scientifique. Un second argument en faveur de ce croisement méthodologique repose sur la structure commune des modèles produits par ces deux domaines. En effet, les modèles fonctionnels et les modèles de données relationnels sont constitués d'une structure similaire, des objets présentant des liens et interactions entre eux et leur environnement, qui rend alors opportun le passage théorique d'un modèle à l'autre. En particulier, le choix d'un modèle relationnel semble tout indiqué car les systèmes techniques urbains peuvent être aisément vus comme une collection d'objets caractérisés par un ensemble d'attributs et entretenant des relations. La notion d'algèbre relationnelle est également un instrument puissant et adapté dans cette étude pour la réalisation d'opérations sur les données.

Plusieurs conclusions apparaissent à ce travail de croisement méthodologique qui a abouti à la construction d'une méthodologie hybride et innovante dans la gestion des risques naturels. Tout d'abord, le passage d'une modélisation fonctionnelle à une modélisation relationnelle et inversement nécessite l'apport de l'information géographique pour être théoriquement valide. L'information géographique permet le croisement entre un zonage spatial de l'aléa avec une position spatiale du système de transport guidé, permettant par la même de déterminer de quelle façon le système est impacté : quels composants sont touchés par l'aléa ? Quelle est l'incidence sur d'autres composants qui sont alors touchés indirectement? Ainsi, l'information géographique apparaît comme la condition sine qua non sans laquelle le passage d'un modèle à l'autre est impossible car présentant une lacune théorique. La seconde conclusion principale qu'il est possible de tirer de ce travail de recherche est que l'implémentation pratique de cette transposition théorique est viable et permet bien de concevoir 
un outil d'évaluation de la vulnérabilité d'un système de transport guidé soumis à une inondation. Un algorithme a été conçu pour permettre d'automatiser le modèle de données relationnel, c'est-à-dire de déterminer les éléments du système qui sont impactés par effet en cascade à partir de la défaillance d'un élément initial.

Cette méthodologie innovante issue du croisement de domaines scientifiques différents et permise par l'information géographique confirme pleinement la place de cette dernière dans la gestion des risques naturels et technologiques en milieu urbain. A l'aide de cette méthodologie proposée dans cet article, il est possible d'évaluer la vulnérabilité d'un système technique face à un aléa naturel de façon intégrative. Elle permet en effet de faire évoluer la gestion des risques vers une approche englobante car elle intègre l'aléa, les systèmes urbains et leurs usages, le territoire ainsi que les interactions entre ces entités. Par conséquent, elle s'intègre pleinement dans la notion de résilience urbaine face aux risques naturels. Elle offre justement la possibilité d'un travail multi-échelle, notamment permis par les outils géomatiques, qui trouve pleinement écho dans les questions d'amélioration de la résilience urbaine pour lesquelles l'imbrication des échelles spatiales est un enjeu majeur.

De plus, cette méthodologie n'est ni propre à un système technique urbain ni à un risque naturel. La méthodologie développée, tant dans sa construction théorique que sur ses principes d'implémentation pratique, est potentiellement transposable à d'autres systèmes techniques et services urbains soumis à d'autres aléas naturels. La complémentarité des résultats obtenus sur divers systèmes techniques soumis à divers aléas offre ainsi de nouvelles pistes d'amélioration de la résilience urbaine par l'amélioration concomitante de la résilience de ces systèmes.

\section{Remerciements}

Ces travaux ont été réalisés en partie dans le cadre du Projet National de recherche VILle 10D-VILlE D'IDÉES (Différentes Dimensions pour un Développement urbain Durable et Désirable Décliné Dans une Dynamique «Dessus / Dessous »); http://www.ville10d.fr

\section{Bibliographie}

ANSSI. (2010). Expression des Besoins et Identification des Objectifs de Sécurité - Méthode de gestion des risques. Rapport technique. Paris, Agence nationale de la sécurité des systèmes d'information (ANSSI).

Arnaud A. (2015). Les limites de la cartographie des risques littoraux : des perspectives pour la compréhension de tous. VertigO - la revue électronique en sciences de l'environnement, p. 1-27.

ASCE. (2009). Guiding Principles for the Nation's Critical Infrastructure. Rapport technique. Reston, Virginia, USA, ASCE Critical Infrastructure Guidance Task Committee. Consulté sur http://www.asce.org/uploadedFiles/Issues $\left\{\_\right\}$and $\left\{\bigsqcup_{-}\right\}$Advocacy/Our $\left\{\_\right\}$Initiatives/ Infrastructure/Content $\left\{\_\right\}$Pieces/critical-infrastructure-guiding-principles-report.pdf

Barroca B., Di Nardo M., Mboumoua I. (2013). De la vulnérabilité à la résilience : mutation ou bouleversement? EchoGéo, vol. 24. Consulté sur http://echogeo.revues.org/13439 
28 RIG. Volume $\mathrm{x}-\mathrm{n}^{\mathrm{o}} 3 / 2016$

Barroca B., Serre D. (2013). Behind The Barriers: A Resilience Conceptual Model. S.A.P.I.EN.S, vol. 6, $\mathrm{n}^{\circ}$ April 2014, p. 11. Consulté sur http://sapiens.revues.org/1529

Beck U. (2001). La société du risque. Sur la voie d'une autre modernité. (Aubier éd. ; L. Bernardi, Trad.). Paris.

Bédard Y., Larrivée S. (2007). Spatial Databases Modeling with Pictogrammic Languages. In Encyclopedia of geographic information sciences.

Benslimane S. M., Benslimane D., Malki M., Amghar Y., Gargouri F. (2006). Construction d'une ontologie à partir d'une base de données relationnelle : approche dirigée par l'analyse des formulaires HTML. Architecture.

Beucher S. (2008). Risque d'inondation et dynamiques territoriales des espaces de renouvellement urbain : les cas de Seine-Amont et de l'Est londonien. Géographie, Université Nanterre - Paris X.

Blancher P. (1998). Risques et réseaux techniques urbains (CERTU, Ed.). Lyon.

Bouchon S. (2006). The Vulnerability of interdependent Critical Infrastructures Systems: Epistemological and Conceptual State-of-the-Art. Rapport technique. Ispra - Italie, European Commission - Directorate General Joint Research Centre, Institute for the Protection and Security of the Citizen, Traceability and Vulnerability Assessment Unit. Consulté sur http://scholar.google.com/scholar?hl=en $\{\backslash \&\} \mathrm{btnG}=\operatorname{Search}\{\backslash \&\} \mathrm{q}=$ intitle:The+Vulnerability+of+interdependent+Critical+Infrastructures+Systems+:

+ Epistemological+and+Conceptual+State- $\{\lfloor\}\}$

Brodeur J., Bédard Y., Proulx M.-J. (2000). Modelling geospatial application databases using UML-based repositories aligned with international standards in geomatics. Proceedings of the eighth ACM international symposium on Advances in geographic information systems - GIS '00, p. 39-46. Consulté sur http://neo-listas.udistrital.edu.co:2131/citation.cfm?id= 355274.355280

Bruneau M., Chang S. E., Eguchi R. T., Lee G. C., O’Rourke T. D., Reinhorn A. M. et al. (2003, nov). A Framework to Quantitatively Assess and Enhance the Seismic Resilience of Communities. Earthquake Spectra, vol. 19, no 4, p. 733-752. Consulté sur http://earthquakespectra.org/doi/abs/10.1193/1.1623497

Bucher B., Le Ber F. (2012). Chapitre 1 Introduction. In Développements logiciels en géomatique : innovations et mutualisation, Lavoisier éd., chap. 1. Paris.

Cagno E., Grande O., Trucco P. (2008). Towards an integrated vulnerability and resilience analysis for underground infrastructures. In 3nd resilience engineering international symposium. Consulté sur https://docs.google.com/a/wmu.se/file/d/0B2lfx \{ _\}f4ofuXYTZPd2ZZanlVenc/edit?usp=sharing

Cerbah F. (2008). Learning Highly Structured Semantic Repositories from Relational Databases : The RDBToOnto Tool. In Proceedings of the 5th european semantic web conference (eswc 2008). Tenerife.

Chen S., Tan J., Ray C., Claramunt C., Sun Q. (2008). An integrated GIS-based data model for multimodal urban public transportation analysis and management. In Geoinformatics 2008 and joint conference on gis and built environment: The built environment and its dynamics, vol. 7144 
Chen Y., Yu J., Khan S. (2010). Spatial sensitivity analysis of multi-criteria weights in GISbased land suitability evaluation. Environmental Modelling \& Software, vol. 25, $\mathrm{n}^{\circ} 12$, p. $1582-1591$.

Commission Européenne. (2011). White Paper, Roadmap to a Single European Transport Area Towards a competitive and resource efficient transport system. COM(2011) 144, 28/03/2011.. Rapport technique. Bruxelles, Commission Européenne.

Cutter S. L., Mitchell J. T., Scott M. S. (2000). Revealing the Vulnerability of People and Places: A Case Study of Georgetown County, South Carolina. Annals of the Association of American Geographers, vol. 90, n 4, p. 713-737. Consulté sur http://www.geo.mtu.edu/ volcanoes/06upgrade/Social-KateG/AttachmentsUsed/SpatialDimensionVulnerability.pdf

DCSSI. (2004). Expression des Besoins et Identification des Objectifs de Sécurité - Mémento. Rapport technique. Paris, Direction Centrale de la Sécurité des Systèmes d'Information Secrétariat général de la défense nationale.

Délégation aux Risques Majeurs. (1990). Les études préliminaires à la cartographie réglementaire des risques naturels majeurs (La Documen éd.). Paris.

D’Ercole R., Metzger P. (2009). Vulnérabilité territoriale : une nouvelle approche des risques en milieu urbain. Cybergeo : European Journal of Geography, n 2209, p. 16. Consulté sur http://cybergeo.revues.org/22022

Feizizadeh B., Jankowski P., Blaschke T. (2014). A GIS based spatially-explicit sensitivity and uncertainty analysis approach for multi-criteria decision analysis. Computers \& Geosciences, vol. 64, $\mathrm{n}^{\circ}$ March 2014, p. 81-95.

Fekete A., Tzavella K., Armas I., Binner J., Garschagen M., Giupponi C. et al. (2015). Critical Data Source; Tool or Even Infrastructure? Challenges of Geographic Information Systems and Remote Sensing for Disaster Risk Governance. ISPRS International Journal of GeoInformation, vol. 4, no 4, p. 1848-1869. Consulté sur http://www.mdpi.com/2220-9964/4/ $4 / 1848 /$

Francis R., Bekera B. (2014). A metric and frameworks for resilience analysis of engineered and infrastructure systems. Reliability Engineering and System Safety, vol. 121, p. 90-103. Consulté sur http://dx.doi.org/10.1016/j.ress.2013.07.004

Galland J.-P. (2003). Calculer, gérer, réduire les risques : des actions disjointes? Annales des Ponts et Chaussées, vol. 106, p. 37-44.

Glatron S. (1997). L'évaluation des risques technologiques majeurs en milieu urbain : approche géographique : le cas de la distribution des carburants dans la région Ile-de-France. Thèse de doctorat, Université Paris 1.

Gleyze J.-F. (2005). La vulnérabilité des réseaux de transport dans un contexte de risques. Thèse de doctorat en analyse théorique et epistémologique en géographie, Université $\mathrm{Pa}$ ris VII - Diderot. Consulté sur http://recherche.ign.fr/labos/cogit/pdf/THESES/GLEYZE/ These \{\\}Gleyze\{\\}2002.zip

Gomarasca M. A. (2009). Basics of Geomatics. Springer Netherlands.

Gonzva M., Barroca B., Gautier P.-E., Diab Y. (2015). A modelling of disruptions cascade effect within a rail transport system facing a flood hazard. In 48th esreda seminar on critical infrastructures preparedness: Status of data for resilience modelling, simulation and analysis. Wroclaw. 
30 RIG. Volume $\mathrm{x}-\mathrm{n}^{\mathrm{o}} 3 / 2016$

Gonzva M., Diab Y., Barroca B., Gautier P.-E. (2014). Résilience des systèmes de transport guidé face aux risques naturels. In Congrès lambda-mu 19 de maîtrise des risques et sûreté de fonctionnement, p. 10. Dijon - France.

Gonzva M., Gautier P.-E. (2014). Résilience des systèmes de transport guidé : du risque de vents traversiers vers le risque hydrologique? In 2ème symposium international en géotechnique ferroviaire - georail 2014, p. 407-416. Marne-la-Vallée, France, IFSTTAR.

Gourmelon F., Robin M., Creuseveau J. G., Pennober G., Simao Da Silva A., Affian K. et al. (2006). Contraintes d'utilisation des technologies de l'information géographique pour la gestion intégrée des zones côtières en Afrique. VertigO - la revue électronique en sciences de l'environnement, vol. 7, n 3, p. 1-23.

Joliveau T. (2004). Géomatique et gestion environnementale du territoire. Recherche sur un usage géographique des SIG. Mémoire d'habilitation à diriger des recherches, Université de Rouen.

Kröger W. (2008, dec). Critical infrastructures at risk: A need for a new conceptual approach and extended analytical tools. Reliability Engineering \& System Safety, vol. 93, no 12 , p. 1781-1787. Consulté sur http://linkinghub.elsevier.com/retrieve/pii/ S0951832008000744

La Porte T. M. (2006). Organizational Strategies for Complex System Resilience, Reliability and Adaptation. In P. E. Auerswald, L. M. Branscomb, T. M. La Porte, E. Michel-Kerjan O. (Eds.), Seeds of disaster, roots of response. how private action can reduce public vulnerability, p. 135-153. Cambridge University Press.

Le Moigne J.-L. (1999). La modélisation des systèmes complexes (Dunod éd.).

Lhomme S. (2012). Les réseaux techniques comme vecteur de propagation des risques en milieu urbain-Une contribution théorique et pratique à l'analyse de la résilience urbaine. Thèse de doctorat en géographie, Université Paris-Diderot. Consulté sur http://halshs.archives -ouvertes.fr/tel-00772204/

Lhomme S., Serre D., Diab Y., Laganier R. (2010). Les réseaux techniques face aux inondations ou comment définir des indicateurs de performance de ces réseaux pour évaluer la résilience urbaine. Bulletin de l'Association de géographes français, p. 487-502. Consulté sur http:// hal.ird.fr/docs/00/58/00/25/PDF/Lhomme-Laganier\{\_BAGF.pdf

Malczewski J. (2004). GIS-based land-use suitability analysis: a critical overview. Progress in Planning, vol. 62, no 1, p. 3-65.

Manche Y. (2000). Analyse spatiale et mise en place de systèmes d'information pour l'évaluation de la vulnérabilité des territoires de montagne face aux risques naturels. Géographie, Université Joseph Fourier - Grenoble I. Consulté sur http://yvanbedard.scg.ulaval.ca/ wp-content/documents/these/These $\left\{\backslash_{\_}\right\}$Yannick $\left\{\bigsqcup_{\}}\right\}$Manche.pdf

Manche Y., Villanova-Oliver M., Martin H., Burnet R. (2002). Un système d'information dans le domaine des risques naturels : le projet SIRVA. Revue Internationale de Géomatique, vol. $12, \mathrm{n}^{\circ} 1$, p. pp. 59-76.

Ministère de l'Écologie du Développement Durable et de l'Énergie. (2012). Première évaluation nationale des risques d'inondation, Principaux résultats EPRI 2011. Rapport technique. 
Mitchell T., Harris K. (2012). Resilience : A risk management approach. Rapport technique $\mathrm{n}^{\circ}$ January. London, Overseas Development Institute. Consulté sur http://www.odi.org/sites/ odi.org.uk/files/odi-assets/publications-opinion-files/7552.pdf

Muller-Mahn D. (2012). The Spatial Dimension of Risk: How Geography Shapes the Emergence of Riskscapes. New York. Consulté sur https://books.google.fr/books?id= QYvVTWA60J8C

Noucher M., Fran G., Noucher M., Fran G. (2008). Pas de decision collective sans appropriation individuelle. Enjeux et limites des technologies de l'information géographique au service du "décider ensemble". In Conférence opde 2008 (les outils pour décider ensemble). Québec, Canada.

November V. (2002). Les territoires du risque : le risque comme objet de réflexion géographique. Bern, Peter Lang.

November V. (2006). Le risque comme objet géographique. Cahiers de géographie du Québec, vol. 50, n 141, p. 289-296. Consulté sur http://id.erudit.org/iderudit/014868ar

November V., Penelas M., Viot P. (2008). ń L'effet Lully ż : un territoire à l'épreuve d'une inondation. Cosmopolitiques, $\mathrm{n}^{\circ} 17$ Juin, p. 89-106. Consulté sur http://www.cosmopolitiques .com/sites/default/files/NovemberPenelasViot.pdf

Noyes D., Peres F. (2007). Analyse des systèmes - Sûreté de Fonctionnement. Techniques de l'ingénieur, p. 9.

O'Rourke T. D. (2007). Critical Infrastructure, Interdependencies, and Resilience. BridgeWashington-National Academy of Engineering, vol. 37, nº 1, p. 22-29.

Oslakovic Stipanovic I., Maat H. ter, Hartmann A., Dewulf G. (2013). Risk Assessment Of Climate Change Impacts On Railway Infrastructure. In P. Carrillo, P. Chinowsky (Eds.), Engineering project organization conference. Devil's Thumb Ranch, Colorado. Consulté sur http://www.epossociety.org/EPOC2013/Papers/Stipanovic\{\\}terMaat\{\\}Hartmann\{ _\}Dewulf.pdf

Pigeon P. (2012). Apports de la résilience à la géographie des risques : l'exemple de La Fautesur-Mer. VertigO - la revue électronique en sciences de l'environnement, vol. $12, \mathrm{n}^{\circ} 1$, p. 1-21. Consulté sur http://vertigo.revues.org/12031

Quenault B. (2013a). Du double affrontement ontologique/axiologique autour de la résilience aux risques de catastrophe : les spécificités de l'approche française. VertigO - la revue électronique en sciences de l'environnement, vol. 13, nº 3 . Consulté sur http://vertigo.revues .org/14510

Quenault B. (2013b). Retour critique sur la mobilisation du concept de résilience en lien avec l'adaptation des systèmes urbains au changement climatique. EchoGéo, vol. 24, no 2013, p. 1-19. Consulté sur http://echogeo.revues.org/13403

Quenault B. (2015). De Hygo à Sendai, la résilience comme impératif d'adaptation aux risques de catastrophe : nouvelle valeur universelle ou gouvernement par la catastrophe ? Développement durable et territoires, vol. $6, \mathrm{n}^{\circ} 3$.

Rinaldi S. M., Peerenboom J. P., Kelly T. K. (2001). Identifying, Understanding, and Analyzing Critical Infrastructure Interdependencies. IEEE Control Systems Magazine, vol. 21, $\mathrm{n}^{\circ} 6$, p. 11-25. 
32 RIG. Volume $\mathrm{x}-\mathrm{n}^{\mathrm{o}}$ 3/2016

Robert B., Morabito L. (2009). Réduire la vulnérabilité des infrastructures essentielles. (Lavoisier éd.). Paris.

Royer M. (2009). HAZOP : une méthode d'analyse des risques - Présentation et contexte. Techniques de l'ingénieur, vol. 33, p. 16.

Saurin T. A., Sosa Gonzalez S. (2013). Assessing the compatibility of the management of standardized procedures with the complexity of a sociotechnical system: Case study of a control room in an oil refinery. Applied Ergonomics, vol. 44, no 5, p. 811-823. Consulté sur http://dx.doi.org/10.1016/j.apergo.2013.02.003

Scarwell H.-J., Laganier R. (2004). Risque d'inondation et aménagement durable des territoires (Presses Un éd.). Lille.

Serre D. (2011). La ville résiliente aux inondations - Méthodes et outils d'évaluation. Thèse de doctorat non publiée, Paris-Est.

Serre D. (2015). Concevoir la résilience urbaine : un défi face à des complexités. In EDP Sciences (Ed.), Complexité et désordre - éléments de réflexion. Consulté sur https://halshs .archives-ouvertes.fr/halshs-01250351

Serre D., Douvinet J., Lhomme S., Balsells M., Barroca B. (2016). Résilience des territoires Articuler les méthodes et disciplines pour proposer des améliorations stratégiques. In Cist - en quête de territoire(s) ? / looking for territories?, p. 446-451. Grenoble.

Serre D., Peyras L., Curt C., Boissier D., Diab Y. (2007). Assessment of civil engineering hydraulic infrastructures. Canadian Geotechnical Journal, no 44, p. 1298-1313.

Simon H. A. (1991). Sciences des systèmes, sciences de l'artificiel (Dunod éd.). Paris.

Talon A., Boissier D., Peyras L. (2009). Analyse de risques : Identification et estimation : Démarches d'analyse de risques - Méthodes qualitatives d'analyse de risques. Consulté sur http://www.unit.eu/cours/cyberrisques/etage $\left\{\_\right\} 3\{\backslash\}$ aurelie/co/Etage $\left\{\_\right\} 3\{\backslash$ _\} synthese $\{\lfloor\}$ web.html

Toubin M. (2014). Améliorer la résilience urbaine par un diagnostic collaboratif - L'exemple des services urbains parisiens face à l'inondation. Thèse de doctorat en géographie, Université Paris-Diderot.

Toubin M., Lhomme S., Diab Y., Serre D. (2013). La Résilience urbaine : un nouveau concept opérationnel vecteur de durabilité urbaine? Développement durable et territoires, vol. 3 , $\mathrm{n}^{\circ}$ Mai 2012, p. 1-15.

Toubin M., Serre D., Diab Y., Laganier R. (2012). An auto-diagnosis tool to highlight interdependencies between urban technical networks. Natural Hazards and Earth System Science, vol. 12, no 7, p. 2219-2224. Consulté sur http://www.nat-hazards-earth-syst-sci.net/12/ $2219 / 2012 /$

Ventura C., Juarez Garcia H., Marti J. (2010). Understanding Interdependencies among Critical Infrastructures. In 9th u.s. national and 10th canadian conference on earthquake engineering. Toronto, Ontario, Canada. Consulté sur http://materiales.azc.uam.mx/area/ Estructuras/2260502/CONG\{\\}INT/2010EQConf-001899\{\\}juarez03.pdf

Veyret Y., Charry G., Meschinet de Richemond N. (2004). Risques naturels et aménagement en Europe (A. Colin, Ed.).

Villemeur A. (1997). Sûreté de fonctionnement des systèmes industriels - Fiabilité, facteurs humains, informatisation (Eyrolles éd.). Paris. 
Weichselgartner J. (2004). Changer au rythme des changements: les défis s'adressant à la gestion des risques naturels. In Risques naturels et aménagement en europe, Armand Col éd., p. 212-221. Paris.

Weichselgartner J., Kelman I. (2014). Geographies of resilience: Challenges and opportunities of a descriptive concept. Progress in Human Geography, $\mathrm{n}^{\circ}$ MAY, p. 0309132513518834. Consulté sur http://phg.sagepub.com/content/early/2014/04/ 02/0309132513518834\$backslash\$nhttp://phg.sagepub.com/content/early/2014/04/02/ 0309132513518834.full.pdf

Zwingelstein G. (1996). La maintenance basée sur la fiabilité (Hermes Sci éd.). 